\title{
Multi-point, multi-mission, high-fidelity aerostructural optimization of a long-range aircraft configuration
}

\author{
Rhea P. Liem, ${ }^{*}$ Gaetan K.W. Kenway,* \\ University of Toronto Institute for Aerospace Studies, Toronto, ON, Canada \\ Joaquim R. R. A. Martins ${ }^{\dagger}$ \\ University of Michigan, Department of Aerospace Engineering, Ann Arbor, MI
}

\begin{abstract}
In this paper we present a new robust approach to produce efficient aircraft using numerical optimization. Our focus is on performing a multi-point optimization that considers the performance at multiple operating points simultaneously. The goal is to avoid severe performance degradation at off-design conditions, which typically occurs with a single-point optimization. Specifically, we aim to design a fuel-efficient long-range aircraft configuration. The robustness is introduced by considering hundreds of missions within the operational flight envelope of similar sized aircraft, based on historical data for the actual flight operations. Due to the large computational cost associated with the high-fidelity multidisciplinary analysis, kriging surrogate models are employed to allow thousands of detailed flight analyses to be performed while limiting the number of high-fidelity evaluations. The methodology is demonstrated in a fuel burn minimization problem of a long-range wide-body aircraft configuration.
\end{abstract}

\section{Introduction}

The demand for commercial aviation is expected to continue increasing at an average of $4.8 \%$ annually through the year 2036 [24]. This growth in air traffic will consequently be reflected in the increasing environmental impact, especially in the areas of noise, air quality, and climate change. At present, the aviation sector contributes approximately $3 \%$ to the global anthropogenic carbon emissions [50]. This contribution may increase to $15 \%$ by 2050 , as suggested in the 1999 Intergovernmental Panel and Climate Change (IPCC) report [45]. The International Civil Aviation Organization (ICAO) Environmental Report 2010 predicts an annual increase of 3.0\% to 3.5\% of the global aircraft fuel consumption [24]. Despite its volatility, the average fuel price follows a growth trend that averages $3.5 \%$ a year [25]. These growing environmental concerns and fuel economy have become the key drivers in designing fuel-efficient aircraft. The ICAO Programme of Action on International Aviation and Climate Change targets a $2 \%$ improvement in global fuel efficiency annually until the year 2050 [24].

A considerable amount of research effort is now directed towards minimizing fuel burn in aircraft. Since estimating fuel burn requires a simultaneous consideration of the many aspects of an aircraft (e.g., structural weight and aerodynamic drag), designing a fuel-efficient aircraft should involve the interdependent disciplines very early in the conceptual design stage. As such, a multidisciplinary design optimization (MDO) approach is useful in the design and assessment of novel fuel efficient aircraft configurations. Moreover, MDO can facilitate design space exploration and quantitative assessment of tradeoffs. Early developments of MDO were directed towards addressing problems in aerospace engineering, owing to the inherent complexity and multidisciplinary nature of aircraft [43]. One of the earliest efforts in integrating aerodynamic and structural design was conducted by Haftka [20], who performed an optimization of flexible wing structures while simultaneously constraining strength and induced drag. Grossman $e t$ al. applied MDO to the design of a sailplane wing [18] and later to a transport wing [19].

To achieve a global fuel burn reduction, it is important to consider the aircraft's operating envelope following its actual usage patterns, i.e., the actual missions that the aircraft fly. A detailed mission analysis involving multiple flight conditions can provide a more accurate fuel burn computation for each flight mission. The traditional single-point analysis, which considers only one flight condition [4, 41, 47, 49], has the tendency to produce designs with very good performance at the selected operating condition at the expense of serious performance degradation at most other off-design conditions [12]. Research efforts have ventured to capture the system's complexity more thoroughly by performing multi-point optimization, which considers the performance at multiple operating points simultaneously. Buckley et al. [8] performed a multi-point optimization to obtain an optimum airfoil design for an aircraft. In this process, 18 operating conditions of distinct combinations of Reynolds number, Mach number, and lift coefficient are

\footnotetext{
* Ph.D. Candidate, AIAA Student Member

$\dagger$ Associate Professor, AIAA Senior Member
} 
considered. Those points are differentiated between on-design and off-design operating conditions where the former refers to the operating conditions where the aerodynamics performance needs to be optimized according to specified design objectives, and the latter refers to operating conditions that impose constraints to the optimization. Toal and Keane [55] performed a multi-point aerodynamic design optimization aiming to minimize the weighted sum of drag coefficients at up to four design flight conditions. For some other multi-point optimization examples, see [12, 42].

Despite the success of these previous works on multipoint optimization, there are several shortcomings. The first is related to the analysis. Previous works used 2D analysis or 3D analysis but without using aerostructural analysis. The second shortcoming is the use of arbitrary weights to combine the operating conditions into a single objective. Selecting these weights can be tedious, especially for a large number of design points, and may not accurately represent the actual intent of the design engineer.

In this paper we address both of these shortfalls. The use of 3D high-fidelity analysis enables us to model the underlying physical phenomena more accurately and with less restrictive assumptions. Multidisciplinary aerostructural optimization can make simultaneous modifications to both the aerodynamic shape and structural design, while fully accounting for the effects of changes in one discipline on the other. The second major contribution is the incorporation of historical data of actual missions. Using this information, we can determine how to best weight a multi-point optimization without resorting to manually choosing weights.

One major challenge is the very high computational cost due to computing thousands flight conditions. The computational expense is exacerbated with multidisciplinary analysis and even more so when high-fidelity models are used. Although such evaluations can be done in parallel, the problem complexity we want to solve keeps increasing, so it is advantageous to have an efficient multi-point optimization framework. A computationally tractable procedure to perform the optimization is therefore sought after.

In this research, we seek to employ surrogate models to significantly reduce the computational costs incurred in objective function evaluation in the multi-point optimization setting. Surrogate models can provide representations of the high-fidelity discipline models that are much cheaper to evaluate than the full models. We wish to use these surrogate models to approximate the aerostructural performance in the cruise regime to allow performing complex mission analyses.

To demonstrate this methodology we will consider the aerostructural design optimization of a long-range aircraft configuration. A surrogate model of the flight envelope will allow for full mission simulation and will lead to robust, optimized full-envelope performance.

\section{Method}

To overcome the poor off-design performance of single point optimized design, a common approach is to perform $N$ analysis at multiple operating conditions and to weight each objective, $f_{i}$, according to some user specified weighting functions, $W_{i}$, yielding:

$$
f=\sum_{i}^{N} W_{i} f_{i} .
$$

The designer now must determine appropriate weighting functions that accurately reflect the real-world operations of that design. In this work we propose an alternative method for "weighting" each of the analysis points in a multi-point optimization.

Our goal is to minimize the weighted average fuel burn from the representative flight missions. At this stage, a simplified problem using a linearized objective function is used to demonstrate our proposed approach. The objective function is thus expressed as the sum of the weighted drag at the various flight conditions, $i=1, \ldots, N$, and the weighted structural weight $\left(W_{s}\right)$, as expressed below,

$$
f=\sum_{i=1}^{N} \mu_{i} D_{i}+\lambda W_{s},
$$

where the weights, $\mu_{i}$ and $\lambda$, are the gradients of the weighted average fuel burn with respect to $D_{i}$ and $W_{s}$, respectively. We now have two important questions to address: (1) where in the flight envelope should we evaluate the drag forces, and (2) how to determine the weighting factors required in the evaluation? To answer these questions we look at the aircraft usage patterns in 2011 from the Bureau of Transportation Statistics (BTS) [57], which show how a given model of aircraft is actually used. In this work, we selected the Boeing 777-200ER and included all domestic flights within the United States, as well as international flights that fly to and from the United States. From the BTS database, we can select the payload and range values for the representative missions to compute the weighted average fuel burn. 
Given the payload and range for each mission, it is now possible to estimate the fuel burn for that particular flight by performing a direct numerical integration.

Analyzing the fuel burn of thousands of missions is very expensive, especially when high-fidelity analyses are involved. Therefore, we use surrogate models to approximate the required aerodynamic coefficients $\left(C_{L}, C_{D}, C_{m_{y}}\right)$, derived based on the high-fidelity data. The surrogate models are constructed on a four-dimensional space of Mach number, angle of attack, altitude, and tail rotation angle. Surrogate modeling techniques can be classified into two main categories, namely the physics-based method and the data-fit or black-box method [2]. The former requires knowledge of the governing equations of the system and is an intrusive method, whereas the latter is computed using interpolation or regression of data generated by solving the high-fidelity model at a set of sample points [60]. The sample points are often generated using a design of experiments (DOE). In this research, we only consider data-fit surrogate models, more specifically the kriging approximation technique [39, 48]. The number of required high-fidelity function calls is therefore equal to the number of sample points required to construct the surrogate model. The cost of constructing and using surrogate models typically increases with the desired accuracy. However, this cost can be considered negligible when compared to the cost of high-fidelity computations. Realizing the importance of a good sample selection in the quality of kriging models, we will also explore a sampling selection procedure based on the actual distribution of operating points.

The procedure to use kriging models and mission analysis algorithm to select the flight conditions $i=1, \ldots, N$, and compute their corresponding weights, $\mu_{i}$ and $\lambda$, is outlined below and its XDSM (eXtended Design Structure Matrix) diagram [34] is shown in Figure 1. The black and shaded grey lines in the XDSM diagram represent process and data flows, respectively.

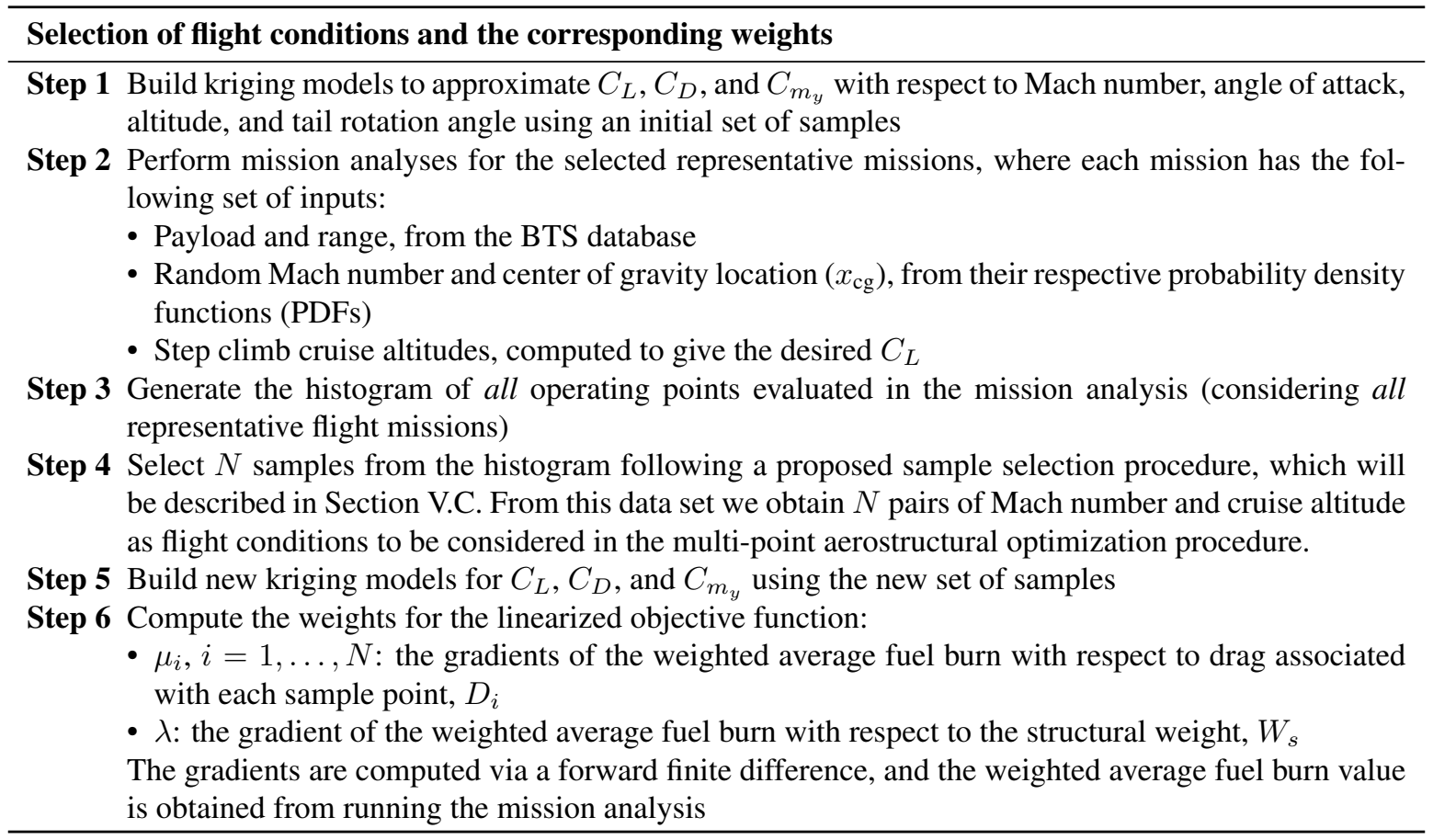

Figure 2 illustrates the XDSM diagram of the multi-point aerostructural optimization performed in this research. The design variables for this optimization, $\mathbf{x}$, include the global variables, $\mathbf{x}_{\text {global }}$, aerodynamic variables, $\mathbf{x}_{\text {aero }}$, and structural thickness variables, $\mathbf{x}_{\text {struct }}$. At each optimization iteration, a multidisciplinary analysis (MDA) performs a high-fidelity aerostructural analysis at each flight condition (Mach, Altitude) ${ }_{i}$ to compute the corresponding drag $D_{i}$. The structural weight, $W_{s}$, is evaluated directly from the structural model. The linearized objective function (2) is then computed using the weights that have previously been obtained and are fixed throughout the optimization procedure, $\mu_{i}$ and $\lambda$. The flight conditions and the weights are outputs from the procedure shown in Figure 1.

\section{Solution Method}

This section outlines the components of the aerostructural optimization performed in this research. We present the multidisciplinary solver that performs the coupled CFD and CSM analyses, followed by a description of mission analysis to compute the amount of fuel burned during a mission. 


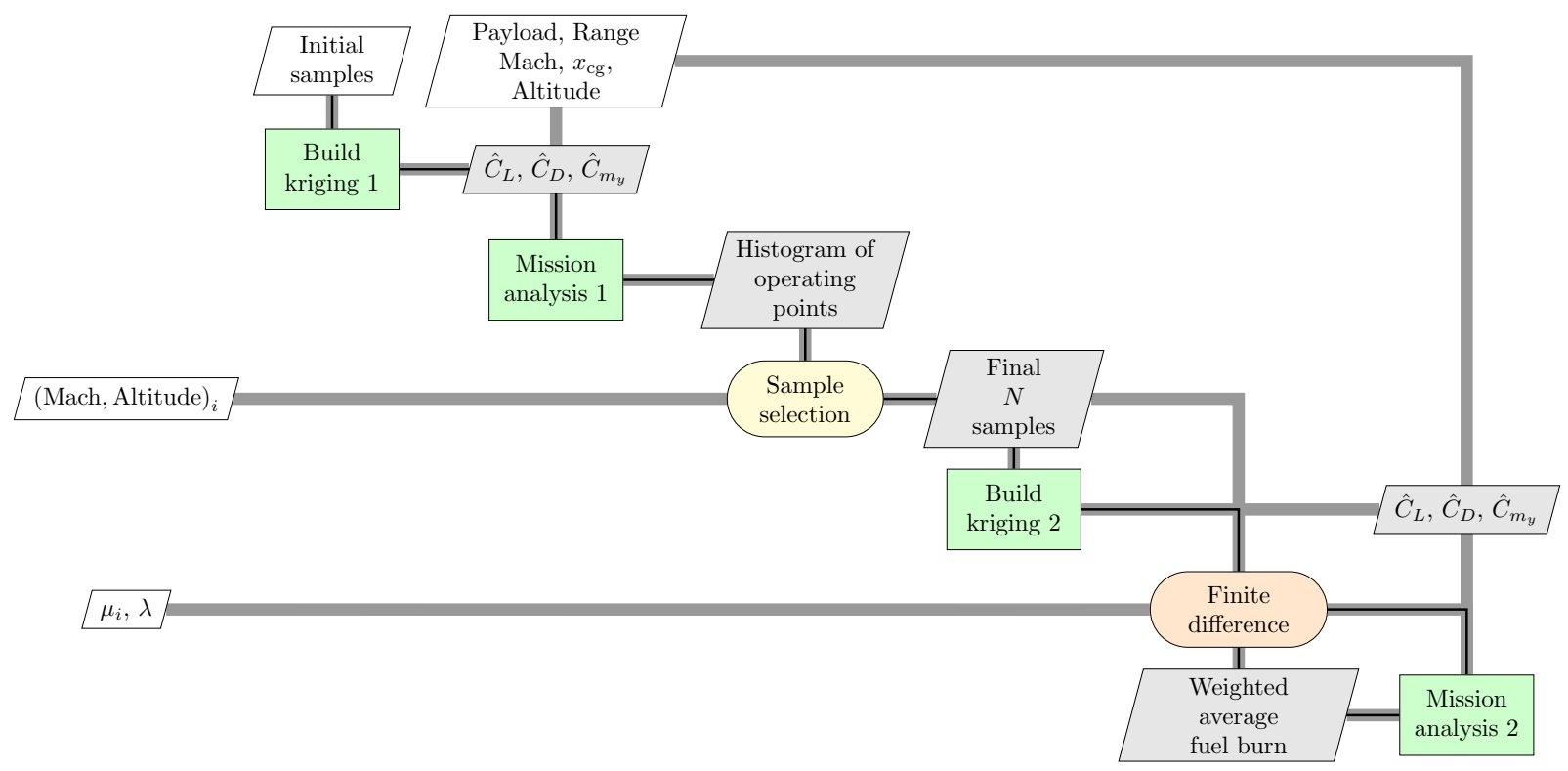

Figure 1: An overview of the mission analysis block to produce the weights $\mu_{i}, i=1, \ldots, N$ and $\lambda$ required for the objective function calculation.

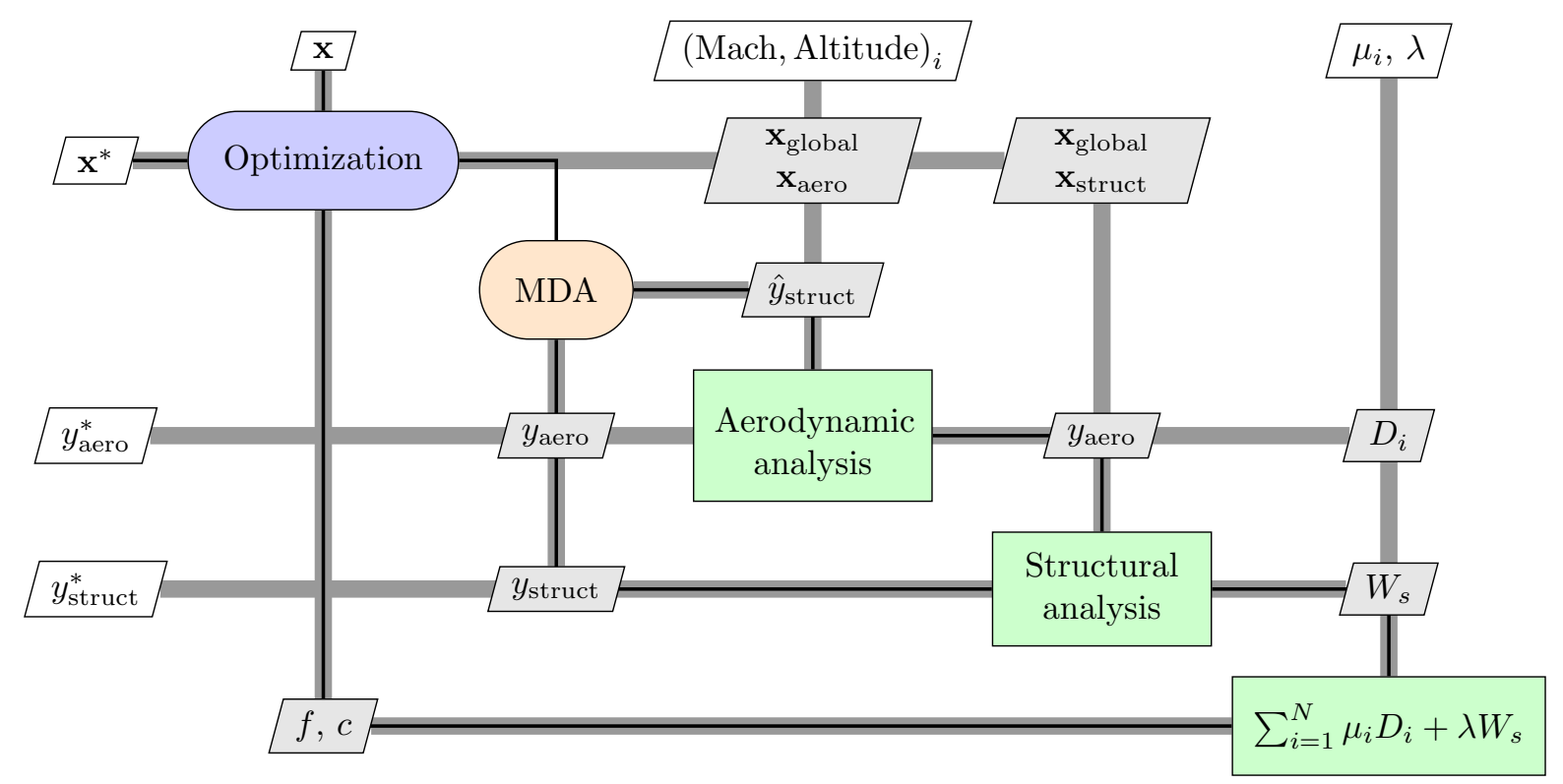

Figure 2: An overview of the proposed multi-point aerostructural optimization approach with linearized objective function. 


\section{A. Aerostructural Analysis}

To evaluate the surrogate modeling technique for use in high-fidelity optimization we employ an aerostructural analysis method developed previously by the authors [31]. Most large commercial aircraft operate in the transonic flight regime during cruise. Therefore, at a minimum, we must solve the Euler equations to accurately predict the wave drag. The CFD solver used in this work is SUmb [58], which solves the Euler equations on multi-block structured grids. The finite element solver is the Toolkit for the Analysis of Composite Structures (TACS) [29]. Critical to the success of this work is the coupled adjoint method that allows us to compute gradients of multidisciplinary functions of interest. For more information on the coupled adjoint technique see Kenway et al. [31].

\section{B. Mission analysis}

The mission analysis is performed to compute the fuel burn of a given flight. The flight mission is divided into five segments: takeoff, climb, cruise, descent, and landing, as illustrated in Figure 3. The takeoff and zero-fuel weights are denoted as $W_{\mathrm{TO}}$ and $W_{\mathrm{ZF}}$, respectively. The zero-fuel weight comprises the operating empty weight and payload (passengers, freight, and airmail). The cruise segment is assumed to include step climbs when the cruise range is longer than $1000 \mathrm{~nm}$. To compute the amount of fuel burned during a mission, we use simple fuel fractions for takeoff, climb, descent, and landing segments. The fuel fraction, $\zeta$, refers to the amount of fuel burned in the segment as a fraction of the segment's initial weight. The general equation to find the segment's final weight given its initial weight and fuel fraction is thus,

$$
W_{f}=(1-\zeta) W_{i},
$$

where $W_{i}$ and $W_{f}$ refer to the segment's initial and final weight, respectively. This equation is used to compute $W_{1}$ given $W_{\text {TO }}$ and $\zeta_{\text {TO }}, W_{2}$ given $W_{1}$ and $\zeta_{\text {climb }}, W_{4}$ given $W_{3}$ and $\zeta_{\text {descent }}, W_{\mathrm{ZF}}$ given $W_{4}$ and $\zeta_{\text {landing. The amount of }}$ fuel burned during the cruise segment, $W_{2}-W_{3}$, is computed by performing direct numerical integration. The fuel fraction values used in this work are listed in Table 1.

\begin{tabular}{lcccc}
\hline Segment & Takeoff & Climb & Descent & Landing \\
\hline Fuel fraction, $\zeta$ & 0.002 & 0.015 & 0.003 & 0.003 \\
\hline
\end{tabular}

Table 1: Fuel fraction values.

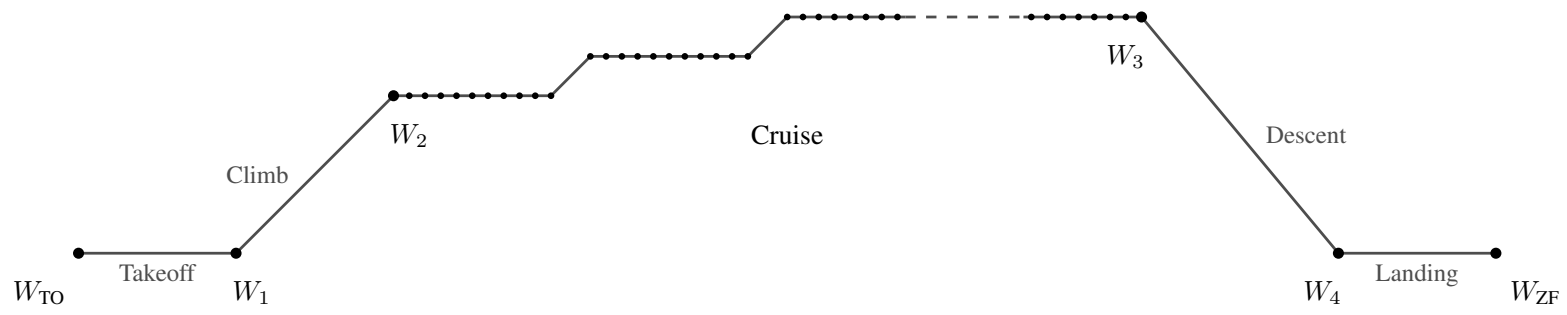

Figure 3: Flight mission diagram

The fuel burn computation for each flight mission needs to make sure that the mission payload and range are satisfied. To compute fuel burn, we first define the thrust specific fuel consumption (TSFC), denoted as $c_{T}$, as:

$$
c_{T}=\frac{\text { Weight of fuel burned per unit time }(N / s)}{\text { Unit Thrust }(N)} .
$$

TSFC is a property of the aircraft engine. The rate of reduction of aircraft weight can thus be computed as,

$$
\frac{d W}{d t}=-c_{T} T
$$

where $W$ and $T$ denote aircraft weight and thrust, respectively. Now we can express range, $R$, as a definite integral of flight speed, $V$, over a certain time interval,

$$
R=\int_{t_{1}}^{t_{2}} V d t
$$


Substituting (5) to (6) lets us define the flight range given the specific range, i.e., the range per unit weight of fuel $\left(-V / c_{T} T\right)$, using the following expression,

$$
R=\int_{W_{2}}^{W_{3}}-\frac{V}{c_{T} T} d W,
$$

where $W_{2}$ and $W_{3}$ refer to the weights at the start and the end of cruise, respectively. Approximating the above range equation by numerical integration, we obtain the following arithmetic expression,

$$
R \approx \sum_{n=1}^{N_{\text {interval }}} \frac{V^{(n)}}{c_{T} T^{(n)}}\left(W_{i}^{(n)}-W_{f}^{(n)}\right),
$$

where $n$ refers to the points $n=1, \ldots, N_{\text {interval }}$ used to integrate the cruise segment of a particular mission. The initial and final weights of the interval $n$ are denoted by $W_{i}^{n}$ and $W_{f}^{n}$, respectively. In order to find the takeoff weight required for the desired cruise range, we iterate over different values of takeoff weight systematically via the secant method that drives the difference between the range obtained at the current iteration and the target range to zero. This procedure is expressed as (9), where $k$ denotes the iteration index. We set the convergence tolerance for this search algorithm to $10^{-8}$.

$$
\begin{gathered}
W_{\mathrm{TO}}^{(k)}=W_{\mathrm{TO}}^{(k-1)}-f\left(W_{\mathrm{TO}}^{(k-1)}\right) \frac{W_{\mathrm{TO}}^{(k-1)}-W_{\mathrm{TO}}^{(k-2)}}{f\left(W_{\mathrm{TO}}^{(k-1)}\right)-f\left(W_{\mathrm{TO}}^{(k-2)}\right)}, \\
f\left(W_{\mathrm{TO}}^{(k)}\right)=R\left(W_{\mathrm{TO}}^{(k)}\right)-R_{\text {target }}
\end{gathered}
$$

When $W_{\mathrm{TO}}$ and $W_{\mathrm{ZF}}$ are known, the initial and final cruise weights ( $W_{2}$ and $W_{3}$ ) can be easily calculated using (3) and the specified fuel fraction values. With these two values, we can perform direct numerical integration to evaluate the cruise range. First, we determine the number of cruise subsegments by assigning a subsegment for each $1000 \mathrm{~nm}$ of cruise range. Next, we divide the amount of fuel burned during cruise equally to those subsegments. The altitude at each step is computed such that it corresponds to a target lift coefficient, which is achieved by performing the secant method. The maximum altitude is set to $41000 \mathrm{ft}$. The relation of flight altitude and $C_{L}$ is given by the lift equation,

$$
L=\frac{1}{2} \rho S_{\text {ref }} V^{2} C_{L},
$$

where $S_{\text {ref }}$ is the reference wing area. The atmospheric density $(\rho)$ and speed of sound, which determines flight speed $(V)$ at a given Mach number, are computed using the standard atmospheric model, as functions of cruise altitude. As we assume a steady level flight, we set the lift force to be equal to the initial weight at each subsegment.

Now we use (8) to evaluate the cruise range. Each cruise subsegment (step) is further divided into 10 intervals, as illustrated in Figure 3, where each cruise interval burns an equal amount of fuel. We set $N_{\text {interval }}$ in (8) to be equal to the number of subsegments times the number of intervals per subsegment. The initial and final weights for each subsegment can easily be found given $W_{2}, W_{3}$, and $N_{\text {interval }}$. We only need $T^{n}$ to complete the evaluation. With the assumption of a steady level flight, we set $D^{n}=T^{n}$, and $L^{n}=W_{i}^{n}$. We can compute $D$ by performing flow solutions while iterating the angle of attack until the desired lift is achieved. To ensure that the flight is at trim condition, we constrain the pitch moment to be zero, $C_{m_{y}}=0$. As mentioned in Section II, we use a surrogate model to approximate $C_{m_{y}}$, which is derived based on the high-fidelity aerostructural analysis. These analyses assume a fixed center of gravity location. Therefore, we add a deviation of pitching moment to accommodate the varying center of gravity locations of the different missions,

$$
\Delta M_{y}=W \cdot g \cdot\left(x_{\mathrm{cg}}-x_{\mathrm{ref}}\right),
$$

where $x_{\text {ref }}$ refers to the location of reference point used to compute pitch moment. This leads to a correction on the pitching moment coefficient,

$$
\Delta C_{m_{y}}=\frac{\Delta M_{y}}{\frac{1}{2} \rho S_{\mathrm{ref}} V^{2} \cdot \mathrm{MAC}}
$$

We use a Newton method to search for the angle of attack $(\alpha)$ and tail rotation angle $(\eta)$ that satisfy these two conditions simultaneously. The general equation for Newton's search algorithm is

$$
\mathbf{x}^{(k)}=\mathbf{x}^{(k-1)}-\mathbf{J}^{-1} \mathbf{f}\left(\mathbf{x}^{(k)}\right),
$$


where $k$ is the iteration index. The function $\mathbf{f}$ is to be driven to zero and $\mathbf{J}$ is the corresponding Jacobian. In this search procedure, the values for $\mathbf{x}, \mathbf{f}$, and $\mathbf{J}$ are

$$
\mathbf{x}=\left[\begin{array}{c}
\alpha \\
\eta
\end{array}\right], \quad \mathbf{f}=\left[\begin{array}{c}
C_{L}-C_{L_{\text {target }}} \\
C_{m_{y}}+\Delta C_{m_{y}}
\end{array}\right], \quad \mathbf{J}=\left[\begin{array}{cc}
\frac{\partial C_{L}}{\partial \alpha} & \frac{\partial C_{L}}{\partial \eta} \\
\frac{\partial C_{m_{y}}}{\partial \alpha} & \frac{\partial C_{m_{y}}}{\partial \eta}
\end{array}\right]
$$

The required number of flow solutions for one flight mission is thus equal to the number of cruise intervals times the number of secant method iterations times the number of Newton search iterations, which can easily reach a few hundred flow solutions. This number grows further when multiple missions are considered. The required computational cost and time quickly becomes intractable, especially when high-fidelity models are used. Using surrogate models to approximate $C_{L}, C_{D}$ and $C_{m_{y}}$, as done in this research, can substantially reduce the computational cost. Moreover, we can directly use the analytical gradients of kriging models, to compute the required Jacobian in the Newton's search algorithm. By doing so, we eliminate the needs to compute high-fidelity gradients in the mission analysis.

\section{Surrogate modeling}

A brief overview of the available surrogate modeling methods, and their applications in aerospace engineering are presented here, followed by a more detailed description of the kriging technique, which was selected for this research.

\section{A. Overview}

The demand for the level of fidelity and complexity that is needed to address real-world engineering problems keeps increasing, outpacing the exponential growth in computing power and the advancement in algorithm [51]. Therefore, simpler approximations of the physical systems are often used as low-cost substitutes for exact evaluations to reduce the computational expenses of analyses $[16,52,53]$. Such approximations are often referred to as surrogate models, response surfaces, metamodels, or models of models $[28,40]$. In the context of optimization, surrogate models are typically used to approximate the objective function either globally (the minimization of predictor approach) or within a pre-specified trust-region (the trust-region approach) [14]. The trust-region method involves solving a sequence of trust-region optimization subproblems [13].

Previous works have demonstrated the effectiveness of surrogate modeling techniques in assisting aerodynamic optimization procedures. The use of a gradient-enhanced kriging method in the supersonic business jet design optimization is demonstrated by Chung and Alonso [10,11]. Toal and Keane [55] minimized the weighted sum of drag coefficients at various flight conditions using cokriging surrogates, where multiple levels of information are used to evaluate the objective function. Koko [33] used a Lagrangian interpolation as a surrogate to model the aerodynamic forces at different points along the flight mission of interest in a trajectory optimization problem aiming to minimize fuel consumption of morphing wingtip devices. Proper-orthogonal decomposition (POD) has been used in airfoil design optimization by various researchers [9, 44, 54]. Zimmermann et al. [64] developed and used POD-subspace restricted least squares model for solving the governing fluid flow equations. Amsallem et al. [3] performed offline precomputations to construct fluid reduced order bases (ROB) and a structural reduced order models (ROM) database for aeroelastic computations. The use of response-surface-based optimizer with sampling refinement strategies for airfoil and wing drag minimization problems was demonstrated by Laurenceau et al. [35, 36].

Despite this extensive use of surrogate models in numerical simulation and optimization procedures, their effectiveness still suffers from the "curse of dimensionality" [5, 32]. Their ability to produce an accurate approximation of the physical system is still limited to problems with relatively low dimensions (less than 50 design variables). Surrogate models that can approximate high-dimensional functions (problems with hundreds or thousands of variables) accurately are still not computationally affordable [6]. Fixed bases radial basis functions (RBFs) and support vector regressions (SVRs) with a fixed kernel (basis function) are suitable for high-dimensional problems but can only handle simple functions and are not very accurate. Moving least-squares (MLS), kriging and parametric RBFs yield more accurate approximations but are still limited to lower-dimensional problems [14].

\section{B. Kriging}

The kriging approximation method is a statistical interpolation method that is constructed by minimizing the mean squared error of the approximation, subjected to the unbiasedness constraint $[48,61]$. The reader is referred to previous works for further details on the derivation and formulation of kriging models [21, 26, 48]. To build a kriging surrogate model, we need sample points of quantity $N_{s}, \mathbf{x}$, and function values evaluated at those sample points, $\mathbf{y}_{s}$. A kriging predictor, $\hat{y}$, at an untried point $\mathbf{x}_{0}$ can then be expressed as,

$$
\hat{y}\left(\mathbf{x}_{0}\right)=\beta+\mathbf{r}_{0}^{T}(\mathbf{x}) \mathbf{R}^{-1}\left(\mathbf{y}_{s}-\boldsymbol{\phi} \beta\right),
$$


where $\mathbf{R}$ denotes the correlation matrix of size $N_{s} \times N_{s}, \phi$ is a matrix of basis functions for the global model $\beta$ (e.g., an $N_{s}$-long vector of ones when a constant global model is used), and $\mathbf{r}_{0}(\mathbf{x})$ denotes the correlation vector between $\mathbf{x}_{0}$ and sample points, as shown below,

$$
\mathbf{r}_{0}^{T}(\mathbf{x})=\left[R\left(\mathbf{x}_{0}, \mathbf{x}^{1}\right), R\left(\mathbf{x}_{0}, \mathbf{x}^{2}\right), \ldots, R\left(\mathbf{x}_{\mathbf{0}}, \mathbf{x}^{N_{s}}\right)\right]^{T} .
$$

The first term of (16) is a global model representing the "function mean", typically modeled as a constant or loworder polynomial function, and the second term is a zero-mean stochastic realization with variance $\sigma^{2}$ and non-zero covariance:

$$
\operatorname{Cov}\left[y\left(\mathbf{x}^{i}\right), y\left(\mathbf{x}^{j}\right)\right]=\sigma^{2} \mathbf{R}\left[R\left(\mathbf{x}^{i}, \mathbf{x}^{j}\right)\right],
$$

where $R\left(\mathbf{x}^{i}, \mathbf{x}^{j}\right)$ is a correlation function between any two sample points $\mathbf{x}^{i}$ and $\mathbf{x}^{j}$. The stochastic term represents localized deviations from the global model, which allows kriging models to perform interpolation between sampled data points. Gaussian exponential correlation is the most commonly used spatial correlation function in kriging model construction, though there are other options such as exponential and cubic spline functions. The formulation for the Gaussian correlation function is shown below,

$$
R\left(\mathbf{x}^{i}, \mathbf{x}^{j}\right)=\exp \left[-\sum_{k=1}^{N_{\mathrm{dv}}} \theta_{k}\left|x_{k}^{i}-x_{k}^{j}\right|^{2}\right],
$$

where $N_{\mathrm{dv}}$ is the problem dimension, $\theta_{k}$ is the unknown correlation parameter associated with the $k$-th design variable used to fit the model, $x_{k}^{i}$ and $x_{k}^{j}$ are the $k$-th components of sample points $\mathbf{x}^{i}$ and $\mathbf{x}^{j}$.

The property of the correlation function ensures that the approximation error of kriging is null at sample points and decreases with distance. A vector of correlation parameters (denoted as $\boldsymbol{\theta}$ ) determines the "strength" of correlation in each direction. These kriging hyperparameters are also referred to as distance weights. Large distance weight values correspond to weak spatial correlation, whereas small values correspond to strong spatial correlation [63]. These kriging hyperparameters are found via a maximum likelihood estimation (MLE) optimization, with the assumption that the approximation errors are normally distributed and that the process variance is stationary, i.e., independent of the locations in the design space. The expression for the optimum correlation parameter, $\boldsymbol{\theta}^{*}$, is given below,

$$
\boldsymbol{\theta}^{*}=\max _{\boldsymbol{\theta}>0}-\frac{\left[N_{s} \ln \left(\sigma^{2}\right)+\ln |\mathbf{R}|\right]}{2}
$$

where $\sigma^{2}$ is defined as follows,

$$
\sigma^{2}=\frac{\left(\mathbf{y}_{s}-\phi \beta\right)^{T} \mathbf{R}^{-1}\left(\mathbf{y}_{s}-\phi \beta\right)}{N_{s}} .
$$

Once the optimum correlation parameters are known, the correlation matrix $\mathbf{R}$ and correlation vector $\mathbf{r}_{0}(\mathbf{x})$ become readily available. The global model can be obtained via a generalized least square method,

$$
\beta=\left(\phi^{T} \mathbf{R}^{-1} \phi\right)^{-1} \phi^{T} \mathbf{R}^{-1} \mathbf{y}_{s}
$$

which completes all the required terms for the kriging predictor, $\hat{y}\left(\mathbf{x}_{0}\right)$. Correspondingly, we can estimate the unknown gradient $\partial \hat{y} / \partial x_{0}^{k}$ at an untried point $\mathbf{x}_{0}$ using the following kriging gradient predictor,

$$
\frac{\partial \hat{y}}{\partial x_{0}^{k}}=\frac{\partial}{\partial x^{k}}\left[\mathbf{r}_{0}^{T}(\mathbf{x})\right]\left(\mathbf{R}^{-1}\left(\mathbf{y}_{s}-\phi \beta\right)\right)
$$

The likelihood function is often too ill-conditioned, causing a failure in finding the optimum hyperparameters. The surrogate model accuracy is thus very sensitive to the selection of the optimization algorithm and sometimes the initial point for the MLE optimization procedure. The likelihood function profile often has the near-optimal ridge property, causing the MLE problem to become difficult to solve, especially when a gradient-based optimizer is used [15]. In this research, the gradient-free Hooke-Jeeves pattern search algorithm is used to solve the MLE optimization problem, as suggested by Lophaven et al. [37], which was also used in other previous works [15, 21, 22]. Pattern search is a direct search routine that is effective to minimize a multidimensional function. Further details of this method can be found in the seminal paper by Hooke and Jeeves [23]; for further discussion and proofs of convergence, refer to the work by Torczon [56]. 


\section{Problem Description}

This section describes the high-fidelity multidisciplinary optimization problem. We present a description of the geometry, the specific selection process for the surrogate model sample locations, as well as the design variables and constraints used in the optimization.

\section{A. Geometry Description}

The initial geometry for the optimization is the common research model (CRM) [59] wing-body-tail configuration. An overview of the configuration is given in Figure 4. It contains design features typical of a transonic, wide-body, longrange aircraft. The configuration has been carefully designed to exhibit good aerodynamic performance across a range of Mach numbers and lift coefficients. This design is a very good aerodynamic starting point for the surrogate modelassisted multidisciplinary optimization since little additional improvement can be made from isolated aerodynamic design modifications alone.

Since the CRM geometry only defines the outer mold line shape, we create a structural model that conforms to the wing outer mold line and is representative of a modern airliner wingbox, as shown in Figure 4.

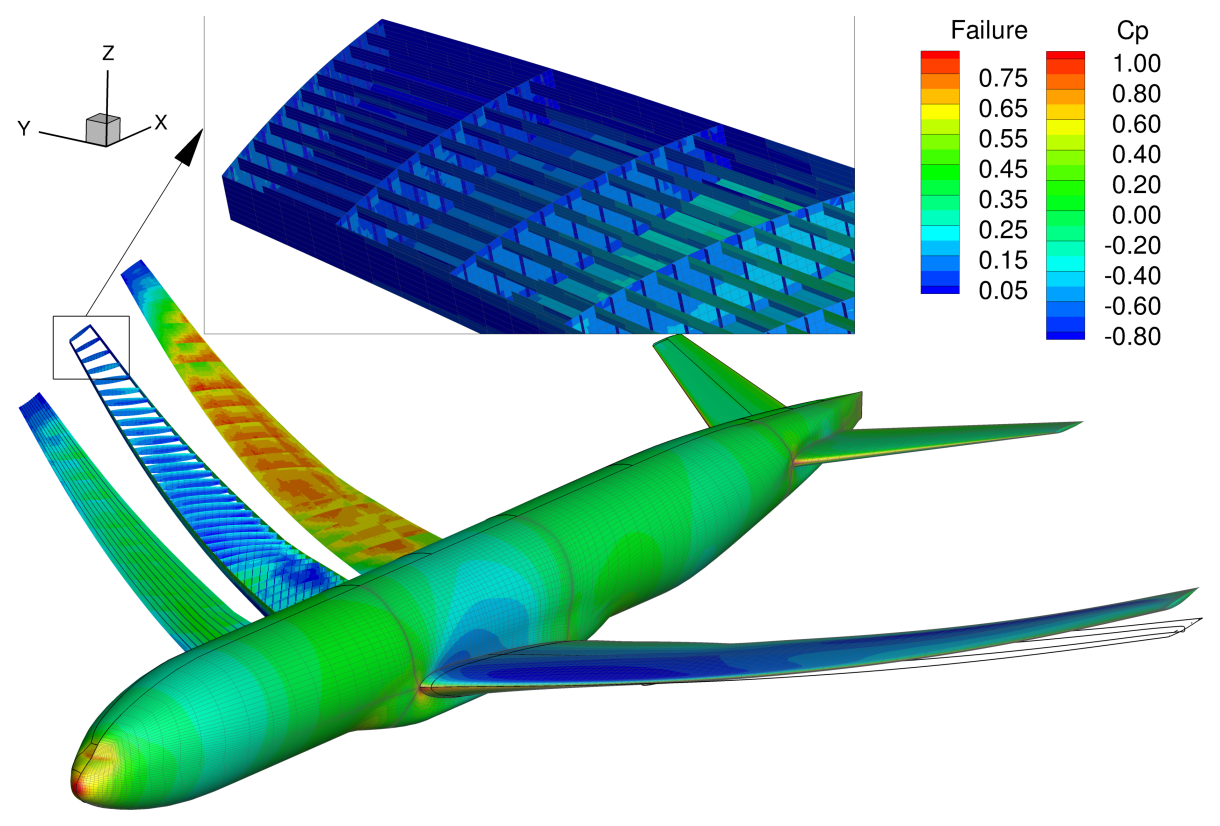

Figure 4: Aerostructural solution for the CRM configuration at Mach=0.85. Outer mold line shows the $1 \mathrm{~g}$ cruise shape with the pressure coefficient distribution (right) and finite element model shows the $2.5 \mathrm{~g}$ deflections and failure criterion (left).

Even with aerodynamic and wing structural models, we need some additional information to perform the required mission analysis. The overall dimensions of the CRM is very similar to the Boeing 777-200ER and the publicly available Boeing 777 Airport Planning Document [7] provides the required additional data. Table 2 lists the key additional parameters used for the optimizations.

\section{B. Flight mission information}

The payload and range distribution of the Boeing 777-200ER from the Bureau of Transportation Statistics' flight database for 2011 is shown in Figure 5. These data represent 101159 flights flying to and from the United States and domestic flights within the United States. From this information we create a set of representative flight missions and assign the corresponding weights to compute the weighted average fuel burn. To select those flights, we create a $25 \times 50$ grid system on a payload-range space and count the number of flight missions that fall on each grid cell. The size of each grid cell is approximately $291 \mathrm{~nm}$ in range by 1 tonne in payload. We take the midpoint of all nonempty grid cells, i.e., cells that contain at least one flight mission, as the representative flight missions. In total, we have 529 representative flight missions. To compute the weights, we normalize the total number of flight missions contained within each cell with respect to the total number of flight missions.

The cruise Mach number and center of gravity locations are not available in the mission database, so we produce values for these parameters using probability density functions (PDFs). The PDFs for these two parameters are 
Table 2: CRM specifications

\begin{tabular}{lrl}
\hline Parameter & Value & Units \\
\hline Nominal cruise Mach number & 0.84 & - \\
Nominal cruise lift coefficient & 0.5 & - \\
Span & 58.6 & $\mathrm{~m}$ \\
Aspect ratio & 9.0 & - \\
Mean aerodynamic chord (MAC) & 7.3 & $\mathrm{~m}$ \\
Reference wing area & 383.7 & $\mathrm{~m}^{2}$ \\
Sweep (leading edge) & 37.4 & $\circ$ \\
Maximum take-off weight (MTOW) & 298000 & $\mathrm{~kg}$ \\
Operational empty weight & 138100 & $\mathrm{~kg}$ \\
Design range & 7725 & $\mathrm{~nm}$ \\
Initial wing weight & 29200 & $\mathrm{~kg}$ \\
Secondary wing weight & 8000 & $\mathrm{~kg}$ \\
Fixed weight & 100900 & $\mathrm{~kg}$ \\
Thrust specific fuel consumption $\left(c_{T}\right)$ & 0.53 & $\mathrm{lb} /(\mathrm{lbf} \cdot \mathrm{h})$ \\
\hline
\end{tabular}

carefully selected to reflect the actual aircraft operation. The missions' Mach number is assumed to have a normal distribution centered at the nominal cruise Mach number, 0.84, with a standard deviation of 0.0067 . The center of gravity location, $x_{\mathrm{cg}}$, is nominally located at a distance of $27.5 \% \mathrm{MAC}$. For each mission it is uniformly varied with $\pm 10 \%$ MAC. Thus, the distributions of these two mission parameters are

$$
\text { Mach } \sim N(0.84,0.006667), \quad x_{\mathrm{cg}} \sim x_{\mathrm{LE}}+U[0.175,0.375] \cdot \mathrm{MAC}
$$

Further randomization is introduced in the cruise altitude. As explained in Section III.B, we assume that the flight missions follow a step climb cruise procedure. A $\pm 1000 \mathrm{ft}$ is then added to the computed altitudes to simulate the variability in assigned altitudes by air traffic control (ATC).

\section{Flight operating points}

We use the samples selected to build kriging models in the second mission analysis block (as shown in Figure 1) as the flight operating points in the cruise condition. Here we first describe the procedure to determine those samples. The flight conditions for the maneuver and stability conditions are described next.

Kriging surrogate models are built for $C_{L}$ and $C_{m_{y}}$ for the Newton search algorithm to find angle of attack $(\alpha)$ and tail rotation angle $(\eta)$ that simultaneously satisfy the desired lift and trim condition at the current Mach number and cruise altitude, as described in Section III.B. Once $\alpha$ and $\eta$ are found, we approximate the corresponding $C_{D}$, also using a surrogate model. The three kriging models $\left(C_{L}, C_{D}\right.$, and $\left.C_{m_{y}}\right)$ are thus built with respect to Mach number, angle of attack, altitude, and tail angle $\left(N_{\mathrm{dv}}=4\right)$. The value ranges for these input parameters are tabulated below. These input parameters are normalized to be between 0 and 1 for the kriging construction and approximation.

\begin{tabular}{ccc}
\hline Input parameter & Lower bound & Upper bound \\
\hline Mach & 0.80 & 0.86 \\
Angle of attack & $0^{\circ}$ & $3^{\circ}$ \\
Altitude & $29000 \mathrm{ft}$ & $41000 \mathrm{ft}$ \\
Tail angle & $-2^{\circ}$ & $2^{\circ}$ \\
\hline
\end{tabular}

Table 3: Value ranges for the surrogate model input parameters.

Kriging is a data-fit surrogate model that relies on sample points to construct the approximations. Selection of samples is thus critical in the prediction quality and accuracy of the kriging model. In this study, we aim to investigate a more methodical way to select kriging samples by looking at the actual distribution of operating points. To do this, we first run a mission analysis using a set of samples, as illustrated in Figure 1. For this run, we use 25 points selected from the input space as specified in Table 3 using the Halton sampling technique, forming the set of "initial samples." The Halton sampling is a deterministic sampling technique that is also a "low-discrepancy" method. Here the discrepancy of a sequence is defined as a measure of the departure of its distribution of points from a uniform distribution, thus it 


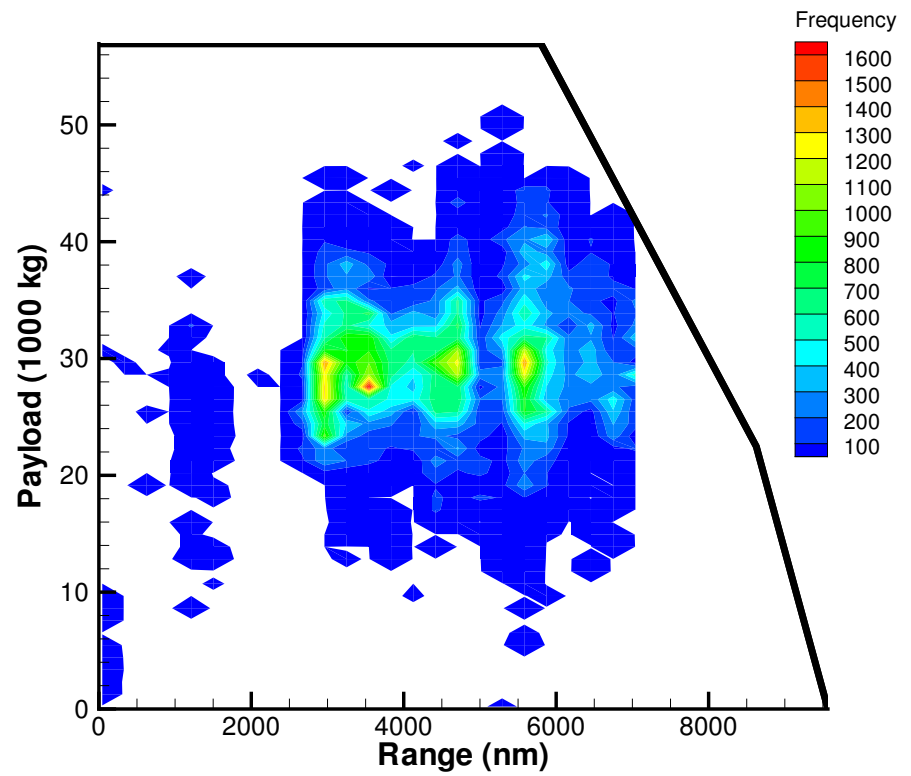

Figure 5: Histogram of 101059 flights for Boeing 777-200ER aircraft during 2011 (Bureau of Transportation Statistics [57]). The payload-range envelope of the Boeing 777-200ER is provided for reference [7].

ensures an even distribution of samples over the design space [27]. Once the samples are determined, we construct the kriging models following the procedure described in Section IV.B. We then run the high-fidelity aerostructural analysis at these sample locations to give us the sample outputs, $\mathbf{y}_{s}$. Note that these analyses only produce pressure drag coefficient. To obtain the total drag coefficient, we add a constant viscous drag coefficient, which is set at 0.0136 . After the required kriging models are built, we run the complete mission analysis.

The total number of operating points evaluated in the mission analysis procedure amounts to $\sum_{m=1}^{N_{\text {missins }}} N_{i_{m}}$, where $N_{\text {missions }}$ is the total number of representative flight missions and $N_{i_{m}}$ is the number of cruise intervals corresponding to the $m$-th mission. In this work, we have a total of 21430 operating points from 529 representative flight missions. These operating points refer to the points in the kriging design space where kriging models are evaluated. Instead of using a continuous function to model the distribution of operating points, a histogram is generated in the fourdimensional grids covering the kriging design space to assist the sample selection procedure. From the distribution of these points, we select the kriging sample points to be more concentrated on higher frequency areas in the design space. By doing so, we ensure better kriging approximations in areas where kriging models are actually evaluated in the mission analysis procedure. This procedure, however, will likely result in some clusters of samples, which is not desirable when kriging is concerned. Small distances between samples can lead to ill-conditioned correlation matrix, as there will be rows that are almost linearly dependent. Thus, we include the sample distance as another criterion in the sample selection procedure.

We start the sample selection by drawing a large number of Halton samples in the normalized space (i.e., where the input parameters are scaled to be between 0 and 1), to give us an initial set that is uniformly distributed in the design space. Those points are then sorted based on their frequencies in a descending order. Points that are less than 0.2 unit distance away from other samples are removed from the list, to prevent clustering. We start with the first Halton point in the remaining list, and gradually add samples by evaluating a "weighted distance" metric, as described in Algorithm 1. The main idea of this metric is to find the points with the highest weights and are as far from other selected points as possible. With this argument, we propose the following metric,

$$
\Phi_{j}=\sum_{s \in \mathcal{S}} d_{j s}\left(w\left(x_{h_{j}}\right)+w\left(x_{s_{s}}\right)\right)
$$

where $\Phi_{j}$ is the weighted distance matrix corresponding to the $j$-th Halton point to be evaluated. $\mathcal{S}$ refers to a set containing all previously selected samples. In this evaluation, the distance between the evaluated point $\left(x_{h_{j}}\right)$ and all 
selected samples $\left(x_{s_{s}}\right), d_{j s}$, are computed. This distance is weighted by the sum of the corresponding weights of the two points in the pair $\left(w\left(x_{h_{j}}\right)\right.$ and $\left.w\left(x_{s_{s}}\right)\right)$. The point with the highest metric is selected to be the next sample. This procedure is repeated until the desired number of samples, $N_{s}$, is achieved. These new samples form the set of "final samples" shown in Figure 1.

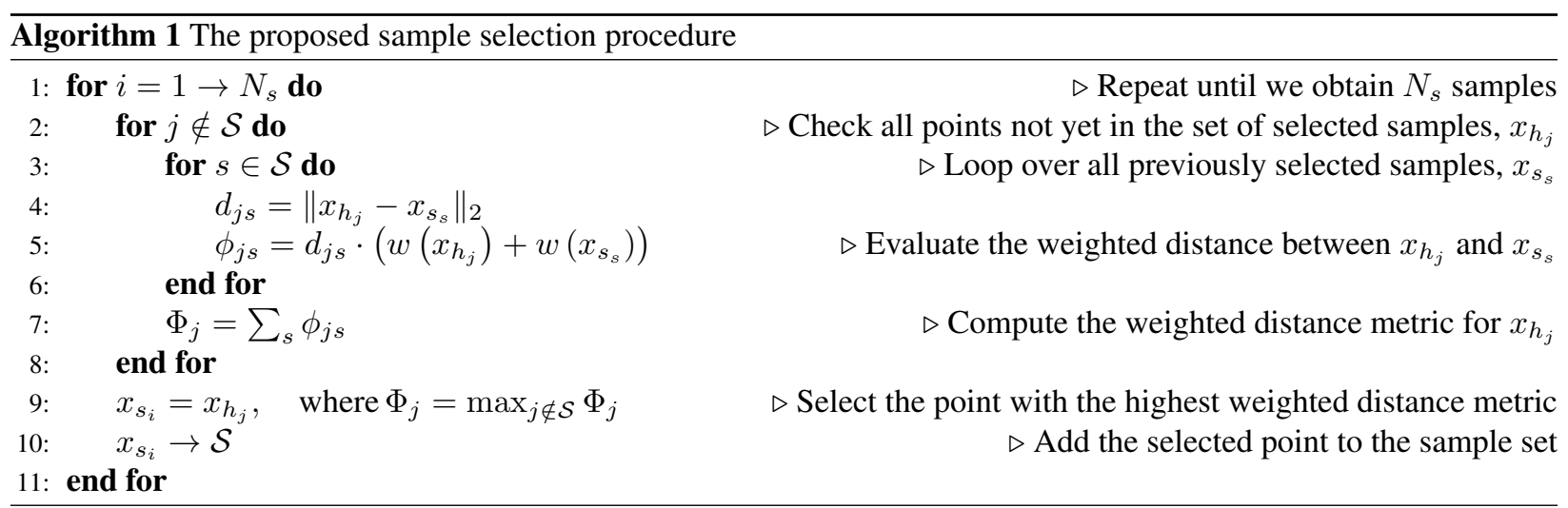

The initial and final samples are compared in Figure 6, which also shows the grid cells, sized with their corresponding weights. For illustration purpose, we only show the design space in a three-dimensional space, by collapsing the tail rotation angle values. The initial samples are shown to cover the design space almost uniformly. The final samples, on the other hand, are concentrated more around the areas where design points have higher weights in the mission analysis. The weighted distance algorithm described above prevents clustering of those sample points, spanning the parameter space in uniform manner, which is essential in building kriging models.

We use the samples selected from Algorithm 1 to be the cruise operating points to evaluate the objective function for the aerostructural optimization procedure. To compute the weighting factors for the objective function (2), the gradients of weighted average fuel burn with respect to drag samples are computed using a forward finite difference.

These selected cruise samples are listed in Table 4. Additionally, two separate maneuver conditions are considered: a $2.5 \mathrm{~g}$ symmetric pull-up maneuver and a $1.3 \mathrm{~g}$ acceleration due to gust. The former maneuver represents a $2.5 \mathrm{~g}$ limit load for the wing structure. The latter maneuver condition represents a gust load on the aircraft in a cruise condition. The purpose of this maneuver condition is explained in the discussion of the optimization problem constraints. A final analysis point is also required for the estimate of the aircraft's static margin. It is possible to estimate the static margin of the deformed configuration using the parameter

$$
K_{n}=-\frac{C_{m_{\alpha}}}{C_{L_{\alpha}}}
$$

Further details on approximating static and dynamic stability derivatives for high fidelity CFD optimization can be found in Mader et al. [38].

\section{Design Variables}

The use of the coupled adjoint method allows us to use hundreds of design variables with little additional cost for gradient evaluations. Table 5 lists all the optimization variables, split between global variables and local disciplinary variables.

We use a free from deformation (FFD) volume approach to parametrize the geometry. Further information on this approach can be found in Kenway et al. [30]. The main wing planform variables, span, sweep, chord and twist are all global variables, as they directly affect the geometry in each discipline. A graphical representation of the geometric design variables, as well as the mesh discretization used for the optimization is shown in Figure 7. Chords are modified at the root, Yehudi break, and tip sections with the remaining sections interpolated. Five twist angles are defined similarly. A single sweep variable sweeps the leading edge of the wing. The shape variables are used to perturb the coefficients of the FFD volume surrounding the wing in the $z$ (normal) direction thus prescribing the cross section thickness and camber.

The structural skin variables are grouped with 18 variables in the span-wise and 3 variables in the chord-wise direction respectively. The stringer variables are grouped similarly. The ribs and rib stiffeners each have 18 variables in the span-wise direction as do each of the leading and trailing edge spars. Since no structure is provided for the CRM model, we must determine a reasonably efficient initial structural model to enable comparisons between the initial and optimized designs. The design of the initial structure is generated by performing a stress-constrained massminimization optimization using fixed aerodynamic loads. 


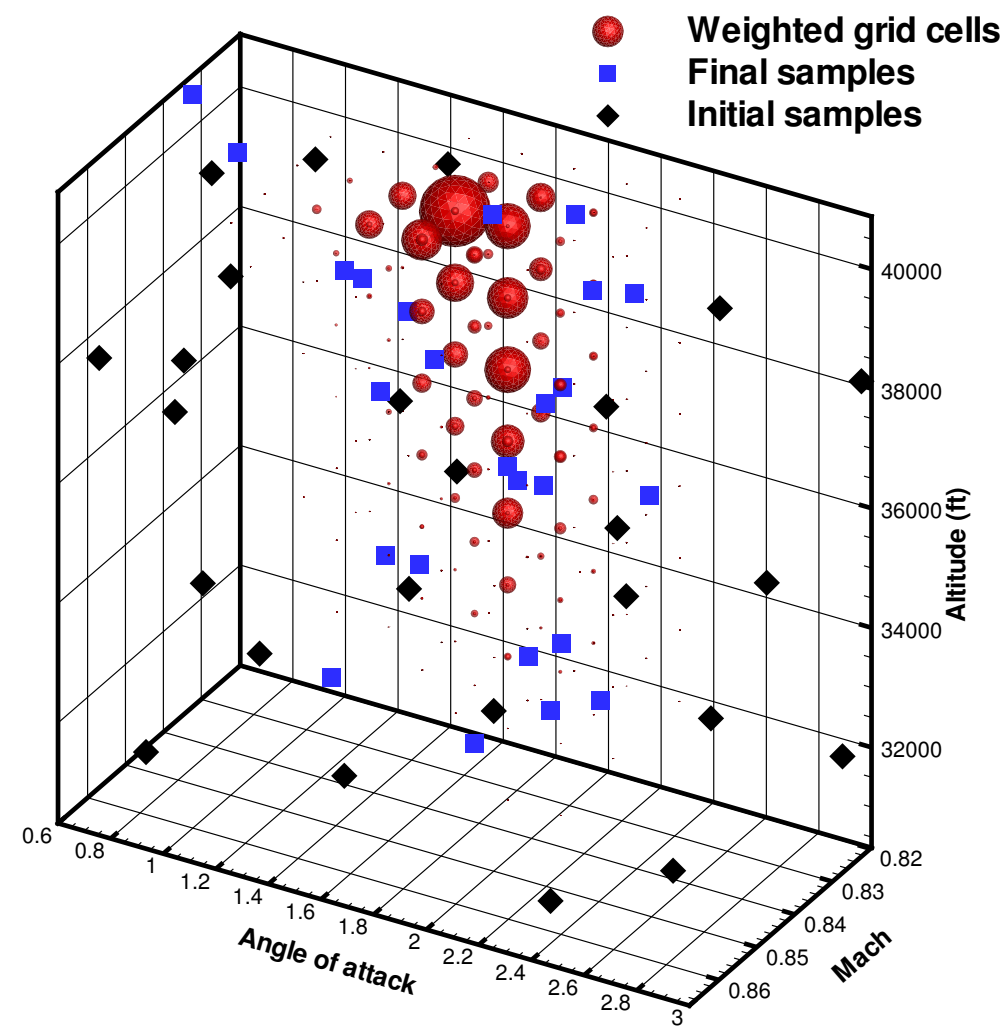

Figure 6: The comparison between initial and final samples. For illustration purposes, we collapse the tail rotation angle values so we can show the points in a three-dimensional space. The distribution of operating points evaluated in the mission analysis is shown with the red spheres, which are sized according to their corresponding weights.

\section{E. Design Constraints}

In this section we describe the constraints that are used for the multidisciplinary optimization. We divide the constraints into three groups: geometric constraints, aerodynamic constraints and structural constraints, as listed in Table 6. The first two geometric constraints $t_{\mathrm{LE}}$ and $t_{\mathrm{TE}}$ are used to constrain the initial wing thickness at $2.5 \%$ and $97.5 \%$ chord, which effectively constrain the leading edge radius and prevents crossover near the sharp trailing edge. The purpose of constraining the leading edge radius is twofold: first, it eliminates excessively sharp leading edges, which are difficult to manufacture, and second, it helps ensure that the high-speed aerostructural optimization does not significantly impact the low speed $C_{L_{\max }}$ performance, which is largely governed by the leading edge radius. We constrain both the projected wing area and internal spar box volume to be no less than their initial values.

Several additional thickness constraints are also enforced. Minimum trailing edge spar height constraints, $t_{\mathrm{TE} \text { spar, }}$, are used over the outboard section of the wing. This constraint is intended to ensure adequate vertical space is available for the flap and aileron attachments and actuators. Maximum thickness constraints $t_{\text {spar box }}$ are used to prevent excessively thick transonic sections with strong shocks causing severe flow separation. Since this phenomenon cannot be modeled with Euler analysis, we use these constraints to help enforce this physical constraint. The final thickness constraint, $t_{\text {tip }}$, is used to ensure the optimization does not produce an unrealistically thin wing tip.

Each cruise condition is constrained to produce the target lift and moment dictated by the sample locations. The static margin of the first cruise condition is constrained to be greater than $25 \%$ with the reference location located at $10 \%$ MAC.

Lastly, we must constrain the structural stresses. We use the Kreisselmeier-Steinhauser (KS) constraint aggregation technique $[46,62]$ to combine many thousand stress constraints into just three. The first KS constraint is for the lower wing skin and stringers, the second is for the upper wing skin and stringers and the third for the spars, ribs and rib stiffeners. The compression members in the upper wing skin use Aluminum 7050 with a maximum allowable stress 
Table 4: Flight operating points

\begin{tabular}{lrrrrrrr}
\hline Group & Identifier & Mach & Altitude (ft) & $\%$ MTOW & $C_{m_{y}}$ & Load Factor & $\mu_{i}$ \\
\hline Cruise & 1 & 0.8379 & 39600 & $69.1 \%$ & -0.116 & 1.0 & 0.1573 \\
& 2 & 0.8405 & 32100 & $74.7 \%$ & -0.138 & 1.0 & -0.0329 \\
& 3 & 0.8593 & 33100 & $90.9 \%$ & -0.003 & 1.0 & -0.0065 \\
& 4 & 0.8490 & 39600 & $61.1 \%$ & -0.174 & 1.0 & -0.0128 \\
& 5 & 0.8592 & 40300 & $63.1 \%$ & -0.085 & 1.0 & 0.0082 \\
& 6 & 0.8231 & 31700 & $84.2 \%$ & -0.077 & 1.0 & 0.0070 \\
& 7 & 0.8307 & 37900 & $57.4 \%$ & -0.078 & 1.0 & -0.0225 \\
& 8 & 0.8282 & 32500 & $83.4 \%$ & -0.171 & 1.0 & 0.0241 \\
& 9 & 0.8421 & 32700 & $93.1 \%$ & -0.161 & 1.0 & 0.0392 \\
& 10 & 0.8413 & 37200 & $64.5 \%$ & -0.138 & 1.0 & 0.1260 \\
& 11 & 0.8203 & 35200 & $76.8 \%$ & -0.121 & 1.0 & 0.0061 \\
& 12 & 0.8437 & 40800 & $64.1 \%$ & -0.163 & 1.0 & 0.1740 \\
& 13 & 0.8430 & 38100 & $61.8 \%$ & -0.005 & 1.0 & 0.1063 \\
& 14 & 0.8466 & 34200 & $87.2 \%$ & -0.045 & 1.0 & 0.0183 \\
& 15 & 0.8473 & 37000 & $79.6 \%$ & -0.186 & 1.0 & -0.0155 \\
& 16 & 0.8180 & 36400 & $63.1 \%$ & -0.032 & 1.0 & -0.0462 \\
& 17 & 0.8272 & 33400 & $64.3 \%$ & -0.057 & 1.0 & 0.0152 \\
& 18 & 0.8578 & 35700 & $77.4 \%$ & -0.025 & 1.0 & 0.0362 \\
& 19 & 0.8373 & 40900 & $39.3 \%$ & -0.012 & 1.0 & -0.0031 \\
& 20 & 0.8326 & 39400 & $71.6 \%$ & -0.182 & 1.0 & -0.0987 \\
& 21 & 0.8251 & 39900 & $59.9 \%$ & -0.083 & 1.0 & 0.1568 \\
& 22 & 0.8526 & 37200 & $81.7 \%$ & -0.094 & 1.0 & -0.0463 \\
& 23 & 0.8265 & 35300 & $65.9 \%$ & 0.007 & 1.0 & 0.0245 \\
& 24 & 0.8589 & 39000 & $79.4 \%$ & -0.080 & 1.0 & 0.0110 \\
& 25 & 0.8380 & 40200 & $46.8 \%$ & -0.124 & 1.0 & 0.0056 \\
\hline 1 & 0.8600 & 20000 & $100.0 \%$ & 0.0 & 2.5 & - \\
& 2 & 0.8500 & 32000 & $100.0 \%$ & 0.0 & 1.3 & - \\
& 1 & 0.8379 & 39600 & $69.1 \%$ & - & 1 & - \\
& & & & & & &
\end{tabular}

Table 5: Design variables

\begin{tabular}{|c|c|c|c|c|c|}
\hline \multicolumn{2}{|c|}{ Global variables } & \multicolumn{2}{|c|}{ Aerodynamic variables } & \multicolumn{2}{|c|}{ Structural thickness variables } \\
\hline Description & Qty & Description & Qty & Description & Qty \\
\hline Span & 1 & Angle of attack & 27 & Upper skin & 54 \\
\hline Sweep & 1 & Tail rotation & 27 & Lower skin & 54 \\
\hline Chord & 3 & & & Upper stringers & 54 \\
\hline Twist & 5 & & & Lower stringers & 54 \\
\hline \multirow[t]{2}{*}{ Shape } & 96 & & & Ribs and stiffeners & 36 \\
\hline & & & & Spars & 36 \\
\hline \multirow[t]{2}{*}{ Total } & 106 & Total & 54 & Total & 288 \\
\hline & & & & Grand total & 448 \\
\hline
\end{tabular}

of $300 \mathrm{MPa}$. The remainder of the primary wing structure uses Aluminum 2024 with a maximum allowable stress of $324 \mathrm{MPa}$.

For the $2.5 \mathrm{~g}$ maneuver condition, the maximum von Mises stress must be below the yield stress, which requires the three KS functions to be less than 1.0. The $1.3 \mathrm{~g}$ maneuver constraint is used to emulate a fatigue criteria for the lower wing skin and stringers that are under tension during normal loading. We limit the stress on the lower wing skin and stringers to be equal to or below $138 \mathrm{MPa}$ for this load condition. The value is selected considering the fatigue stress limit for aluminum 2024. The upper limit for this KS function is 0.42 . The remaining two KS functions for the $1.3 \mathrm{~g}$ maneuver condition retain a the maximum KS value of 1.0. 


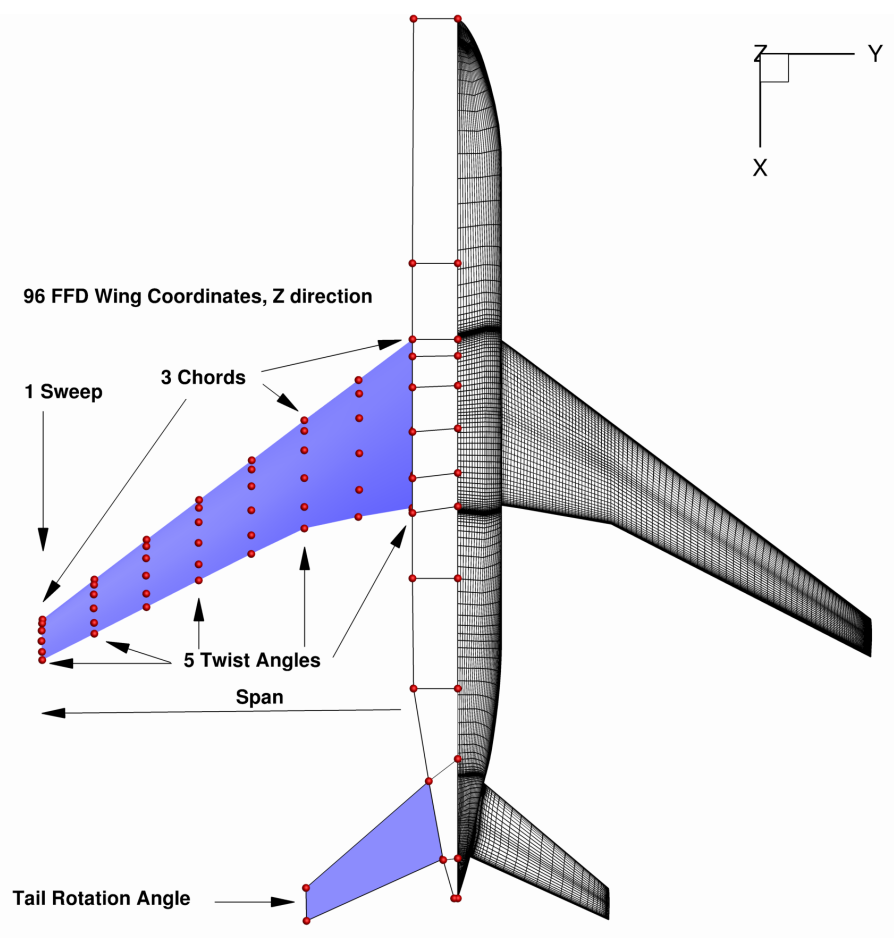

Figure 7: Geometric design variables and FFD volumes (left) and CFD surface mesh (right) for the CRM design optimization problem.

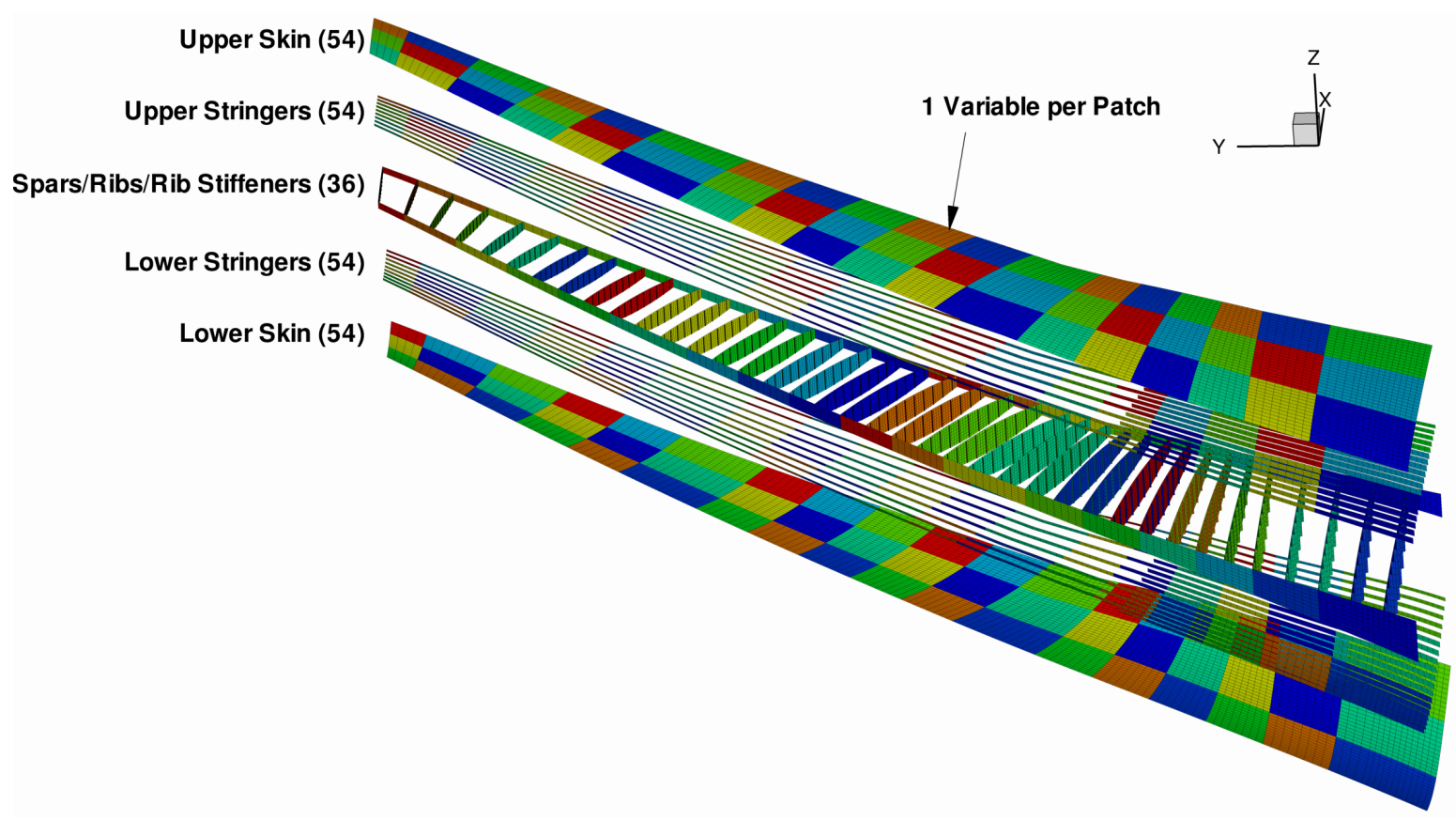

Figure 8: Structural thickness design variable groups.

\section{F. Optimization Algorithm}

Due to the costly nature of multi-point optimization, it is highly desirable to use a gradient-based optimization method to reduce the number of function evaluations necessary to reach a local optimum. The coupled adjoint method allows us to compute gradients of functions of interest with respect to hundreds of design variables very efficiently. For this 
Table 6: Design optimization constraints

\begin{tabular}{|c|c|c|c|c|c|c|c|c|c|c|c|}
\hline \multicolumn{4}{|c|}{ Geometric/target constraints } & \multicolumn{4}{|c|}{ Aerodynamic constraints } & \multicolumn{4}{|c|}{ Structural constraints } \\
\hline \multicolumn{2}{|l|}{ Description } & \multicolumn{2}{|c|}{ Quantity } & \multicolumn{2}{|l|}{ Description } & \multicolumn{2}{|c|}{ Quantity } & \multicolumn{2}{|l|}{ Description } & \multicolumn{2}{|c|}{ Quantity } \\
\hline$t_{\mathrm{LE}} / t_{\mathrm{LE}_{\operatorname{Init}}}$ & $\geq$ & 1.0 & 11 & Cruise: $L$ & $=$ & $L^{*}$ & 25 & $2.5 \mathrm{~g}$ Lower skin: $K S$ & $\leq$ & 1.0 & 2 \\
\hline$t_{\mathrm{TE}} / t_{\mathrm{TE}_{\mathrm{Init}}}$ & $\geq$ & 1.0 & 11 & Cruise: $C_{m_{y}}$ & $=$ & $C_{m_{y}}^{*}$ & 25 & $2.5 \mathrm{~g}$ Upper skin: $K S$ & $\leq$ & 1.0 & 2 \\
\hline$A / A_{\text {init }}$ & $\geq$ & 1.0 & 1 & Maneuver: $L-W$ & $=$ & 0.0 & 2 & $2.5 \mathrm{~g} \mathrm{Rib} /$ spars: $K S$ & $\leq$ & 1.0 & 2 \\
\hline$V / V_{\text {init }}$ & $\geq$ & 1.0 & 1 & Maneuver: $C_{m_{y}}$ & $=$ & 0.0 & 2 & $1.3 \mathrm{~g}$ Lower skin: $K S$ & $\bar{x}$ & 0.42 & 2 \\
\hline$t_{\mathrm{TE} \text { Spar }}$ & $\geq$ & 0.20 & 5 & Static margin: $K_{n}$ & $\geq$ & 0.25 & 1 & $1.3 \mathrm{~g}$ Upper skin: $K S$ & $\leq$ & 1.0 & 2 \\
\hline$t_{\mathrm{Tip}} / t_{\text {Tip }}$ & $\geq$ & 0.5 & 5 & & & & & $1.3 \mathrm{~g} \mathrm{Rib} /$ spars: $K S$ & $\overline{\leq}$ & 1.0 & 2 \\
\hline$M A C-M A C^{*}$ & $=$ & 0.0 & 1 & & & & & & & & \\
\hline$X_{\mathrm{CG}}-X_{\mathrm{CG}}^{*}$ & $=$ & 0.0 & 1 & & & & & & & & \\
\hline$t_{\text {Spar Box }} / t_{\text {Spar Box }_{\text {init }}}$ & $\leq$ & 1.1 & 55 & & & & & & & & \\
\hline \multirow[t]{2}{*}{ Total } & & & 91 & Total & & & 55 & Total & & & 12 \\
\hline & & & & & & & & Grand total & & & 158 \\
\hline
\end{tabular}

work we use SNOPT, an optimizer based on the SQP approach [17].

\section{G. Computational Resources}

The aerostructural optimization is performed on a massively parallel supercomputer [1]. Each function evaluation requires 28 flow solutions: 25 for the cruise conditions, two for the maneuver conditions and one for the stability point. Individual aerostructural solutions are computed in parallel and all flow solutions are computed concurrently. The cruise and stability conditions use 28 aerodynamic processors and 4 structural processors while the maneuver conditions use 45 aerodynamic processors and 4 structural processors. Variable number of processors for the different conditions is required since the maneuver conditions must solve for five adjoint vectors (lift, moment and three KS functions) while the cruise conditions only compute three (lift, drag and moment). The total number of processors used is $931=32 \times 25+32+49 \times 2+1$. The single additional processor at the end is used for the skin friction computation.

To help reduce the computational cost, a relatively coarse 1.2 million cell CFD mesh is used the optimization. Once the optimization was completed, the resulting design was re-analyzed on a 2.1 million cell CFD mesh and values obtained from this solution are presented in the next section. The structural discretization contained approximately 300000 degrees of freedom.

\section{Results}

We now examine the results from the aerostructural optimization. Figure 9 shows the convergence history of the merit function, feasibility and optimality [17].

The optimization was run for 48 hours during which 138 major iterations were completed. The optimality is reduced by approximately 1.5 orders of magnitude, consisting of only a weak local optimum. Unfortunately, due to computational cost considerations, we were not able to continue the optimization to further improve the objective. However, we expect that, given the trend in the merit function, only a small improvement would be achieved if the optimization was to continue. The final design is feasible with respect to all the design constraints.

After the optimized design is obtained, we reconstruct kriging models and run the complete mission analysis with the exact mission parameters as the run corresponding to the initial design. In the new kriging models, we use the same hyperparameters as those used in the kriging models corresponding to the initial design and the new sample inputs and outputs from the optimized design. Using this new model, we extract three drag divergence curves for the optimized design to compare with the initial design. The result is given in Figure 10.

The drag reductions are consistent across the full range of Mach numbers clearly demonstrating the effect of the multi-point optimization algorithm. Both the $C_{L}=0.50$ and $C_{L}=0.55$ curves show a slight drag "bucket" around the nominal cruise Mach number of 0.84, providing performance improvements at these most frequently flown Mach numbers. However, it is not too deep, and due to the multi-point analysis, we do not observe severe performance degradation at off-design Mach numbers. The optimized configuration does exhibit a more rapid drag rise at higher Mach numbers indicating the optimizer has successfully traded this high Mach performance for lower drag in the region centered around the design Mach number. 


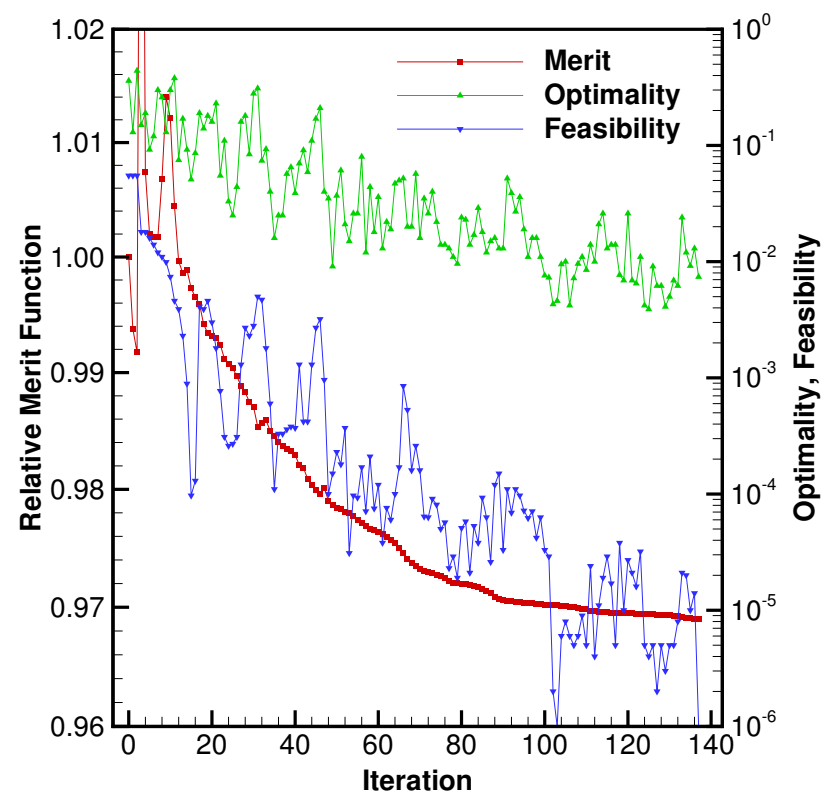

Figure 9: Optimization convergence histories for SNOPT merit function, optimality and feasibility.

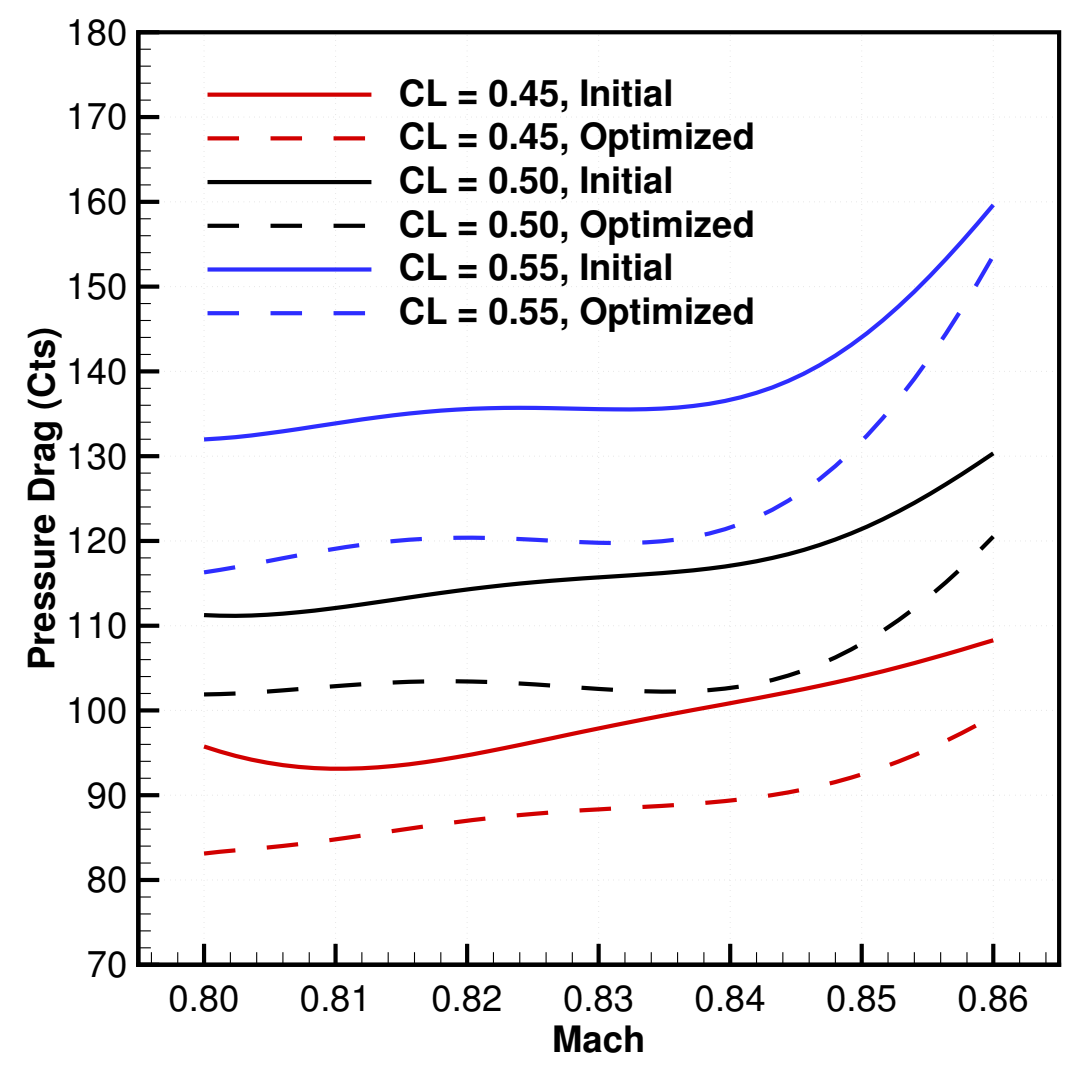

Figure 10: Trimmed drag divergence curves (altitude $=37000 \mathrm{ft}$ ) extracted from kriging model for initial and optimized designs. 
Using the new kriging surrogate models, we recompute the average fuel burn from the 529 sample missions. We observe an $8.8 \%$ reduction in the weighted average fuel burn, from $56905 \mathrm{~kg}$ when the initial design is used, to 51897 $\mathrm{kg}$ with the optimized design. Figure 11 shows the distribution of percent change of fuel burn for individual missions in the payload-range diagram. Higher percentage of fuel burn reduction is observed in the high-payload and highrange region (up to $11 \%$ lower fuel burn). We observe fuel burn increases in some regions corresponding to low cruise range. These regions correspond to the kriging design space that are not very well-sampled (recall that we move the kriging samples to the areas with higher frequency distribution of the operating points). The relatively poor kriging approximation accuracy in these regions partially explains the higher fuel burn, despite the overall decrease in $C_{D}$ used to construct the kriging surrogate model. Additionally, since these short flights contribute very little to the overall fuel burn objective, a significant performance penalty can be incurred for these flights with little effect on the objective function.

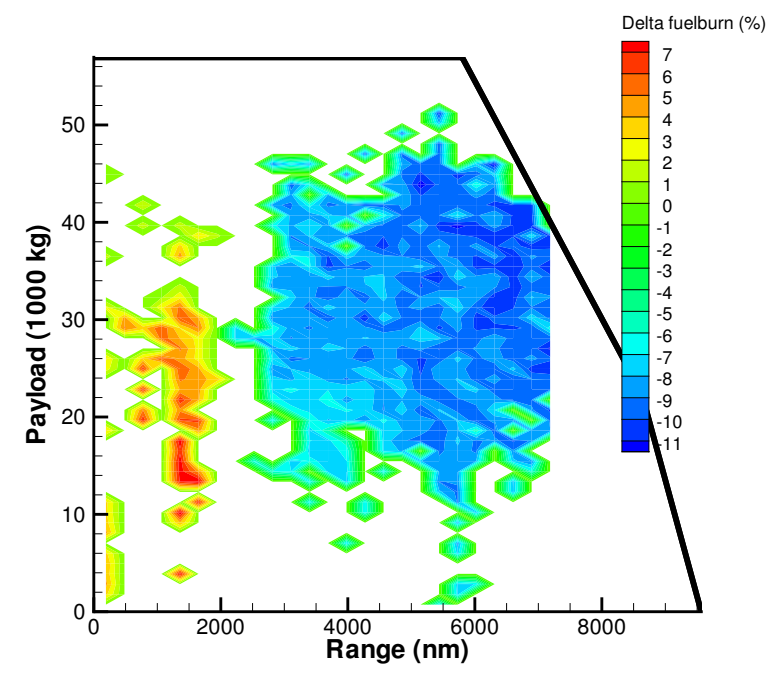

Figure 11: The distribution of percent change of fuel burn for individual missions in the payload-range diagram.

The linearized objective weights, i.e., the gradients of the weighted fuel burn with respect to $D$ at the selected 25 operating points, are also recomputed. The comparison of these weights from the initial and optimized design points is displayed in Figure 12. Most of the values change, which is expected because the aerostructural optimization procedure results in slightly different operating points from the initial design, so the weights are not evaluated at exactly the same points. We do not see any correlation between the change in drag between the initial and optimized designs, and the corresponding change in weights.

Next we examine the overall planform change resulting from the optimization. Figure 13 shows the $C_{p}$ contours on the upper surface of the initial and optimized CRM configurations. Cruise condition 13 is displayed as this sample point most closely matches the design point conditions for the CRM configuration. The most significant change to the design is an approximately $8 \mathrm{~m}$ span extension. Additionally, the root chord and leading edge sweep increased slightly. The total wing area remained at its lower bound, thus the net effect of these changes is an increased aspect ratio and lower taper ratio. The $C_{p}$ contours are reasonably complex as the detailed shape changes are used to reduce the wave drag across a large number of sample points.

Table 7 lists the initial and optimized masses for the key structural components of the wing box. As it happened, the total structural mass remained essentially unchanged, reducing only by $69 \mathrm{~kg}$. The distribution of mass did change slightly but not significantly. The total mass did, however, vary considerably during the course of the optimization. Intermediate values of $\pm 1000 \mathrm{~kg}$ were observed before settling down near the initial starting mass.

Given the rather large span increase, a constant wing area, and the fact that the primary wing box structural mass has remained constant, we may wonder how the failure criteria are not violated on the optimized design. Figure 14 gives us some insight into how the optimized design makes use of the aerostructural tradeoffs and aeroelastic tailoring.

Firstly, the thickness to chord ratio of the optimized configuration has been increased substantially, especially in the critical Yehudi break region of the wing. The thicker wing allows for a more efficient load carrying structure supporting the increased span with essentially the same structural mass. The twist distributions plotted in Figure 14 


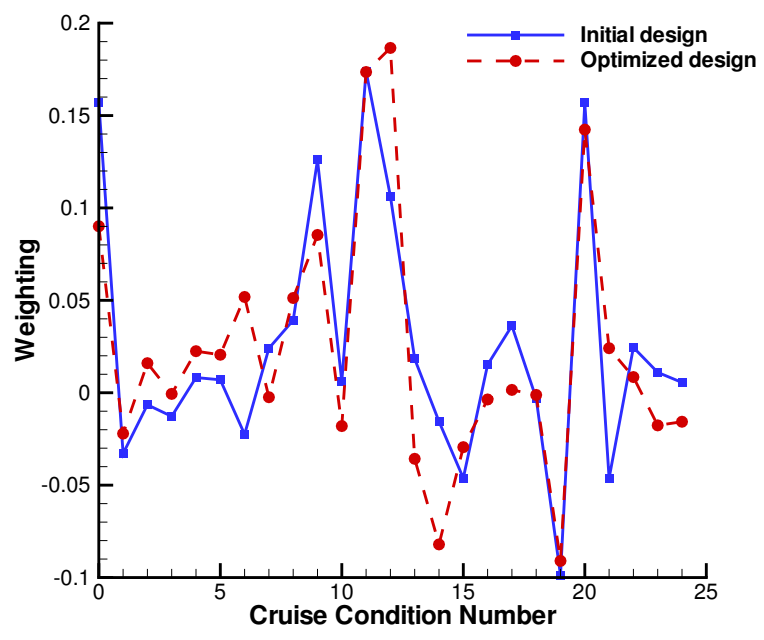

Figure 12: Comparison of linearized objective weights for the initial and optimized design points.

Table 7: Mass breakdown

\begin{tabular}{lrr}
\hline Component & Initial design $(\mathrm{kg})$ & Optimized design $(\mathrm{kg})$ \\
\hline Bottom skin & 6326 & 6160 \\
Bottom stringers & 1027 & 1068 \\
Top skin & 3759 & 3652 \\
Top stringers & 278.7 & 286.5 \\
Rib stiffeners & 22.5 & 27.2 \\
Ribs & 290.4 & 258.3 \\
Front spar & 617.3 & 734.9 \\
Rear spar & 361.7 & 464.4 \\
\hline Total & $\mathbf{1 2 ~ 7 2 1}$ & $\mathbf{1 2 6 5 2}$ \\
\hline
\end{tabular}

are the actual $1 \mathrm{~g}$ twist, not the design or jig twist, thus each cruise condition will have a slightly different twist distribution. The maximum and minimum twist distributions from the 25 cruise conditions are included to highlight the range of twists experienced during cruise. The cruise twist distributions for the initial and optimized configurations are fairly similar, indicating the twist distribution on the CRM geometry was a fairly good starting point. However, the optimized twist for the $2.5 \mathrm{~g}$ maneuver case shows a very significant amount of washout. This effect can be more clearly observed in Figure 15 which shows the cruise and maneuver lift distributions for the initial and optimized configurations.

The lift distribution for the initial cruise condition is highly loaded near the tip, and closer to an elliptic distribution. The higher aspect ratio optimized design is also more highly tapered, which results in a significant reduction in the amount of lift near the tip, even for the cruise condition. More dramatic is the very significant inboard shifting of the lift distribution for the maneuver condition, which reduced the bending moments, resulting in a lighter structure than would otherwise be possible.

We can also examine the thickness distributions and failure load (fraction of von Mises stress to the yield stress) in the initial and optimized design. Since the total mass is almost the same between the two designs we expect only a redistribution of the material, which is seen in Figures 16a and 16c. Since both designs are feasible with respect to the KS failure constraints, we expect a maximum KS value of 1.0 which is seen in both the initial and optimized designs. For the $2.5 \mathrm{~g}$ maneuver condition, only the upper wing skin and stringers are critical, as the lower skin and stringers are dimensioned by the $1.3 \mathrm{~g}$ gust load case. 


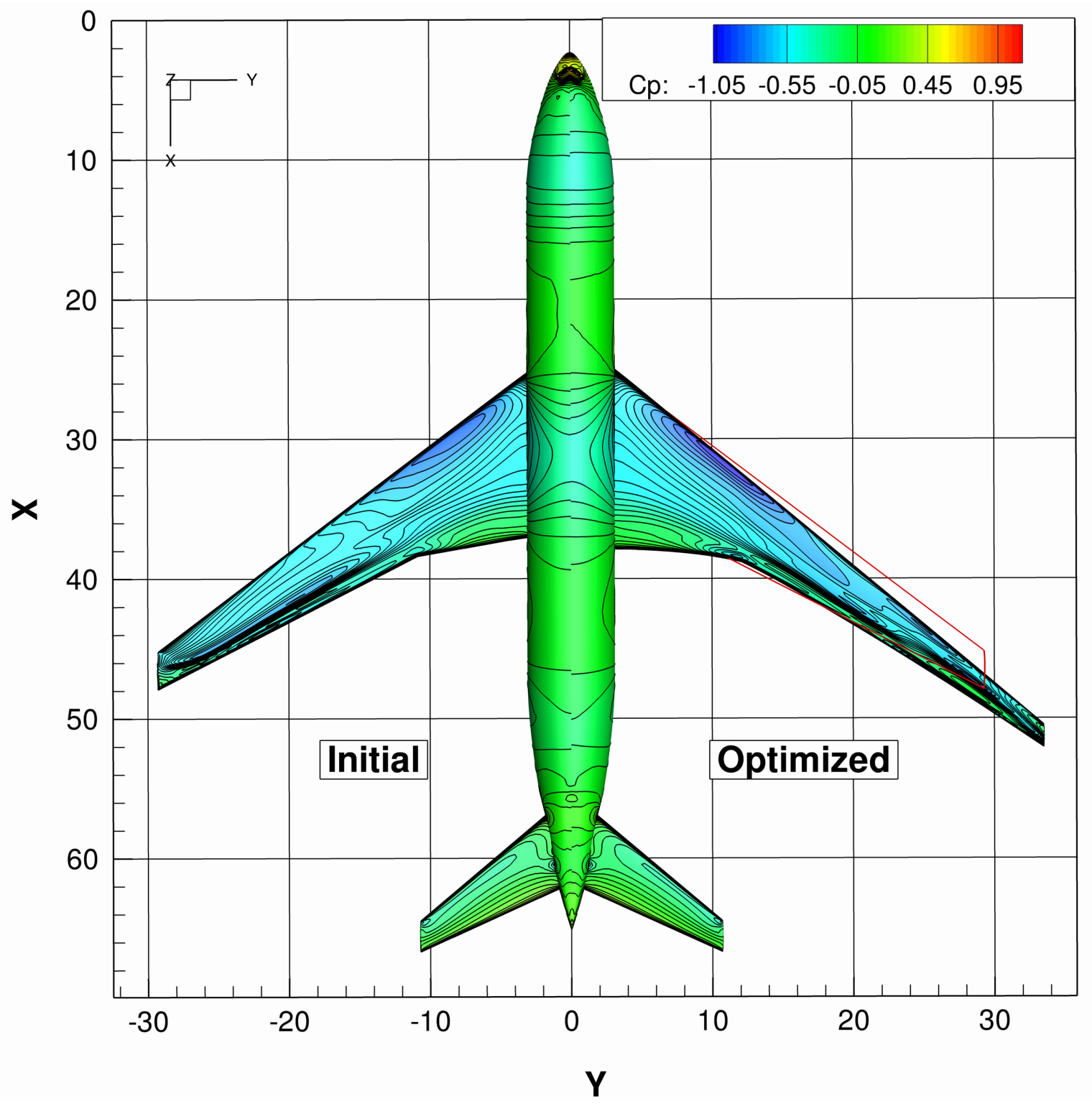

Figure 13: Planform view with $C_{p}$ contours for the initial and optimized designs at cruise condition 13 (Mach number $=0.843$ and altitude $=38100 \mathrm{ft}$ ). The corresponding solution for the initial design (on left) and planform outline (on right), are included for reference.

\section{Conclusions}

A new method for performing multi-point high-fidelity aerostructural optimization of aircraft configurations was presented. The unique attribute of the method is the systematic determination of which points in the flight envelope should be analyzed as well as how much each should contribute to the overall objective.

Our method uses historical data for an aircraft configuration to determine how the aircraft are operated in the real world. This raw data consists of over one hundred thousand flight records, from which, we produced a representative set of 529 missions that accurately reflected the payload-range distribution in actual usage. Next, we select a small set of sample locations based on the distribution of actual operating points of the aircraft to create kriging surrogate models that approximate the aerostructural performance in the cruise regime. Finally, the sensitivity of our objective function, i.e., the weighted average fuel burn over all missions, was computed with respect to the sampled drag forces. These sensitivities formed the weighting factors for the high-fidelity multi-point aerostructural analysis.

The method was tested by using representative long-range aircraft configuration similar to the Boeing 777-200ER 


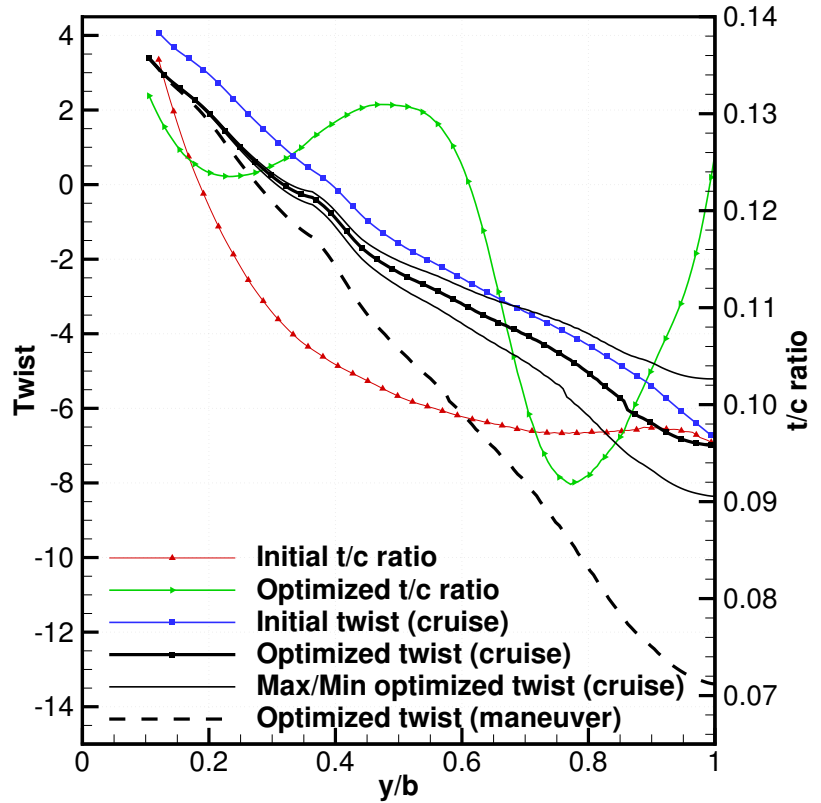

Figure 14: Twist and thickness to chord variation for the initial and optimized designs (cruise condition 13).

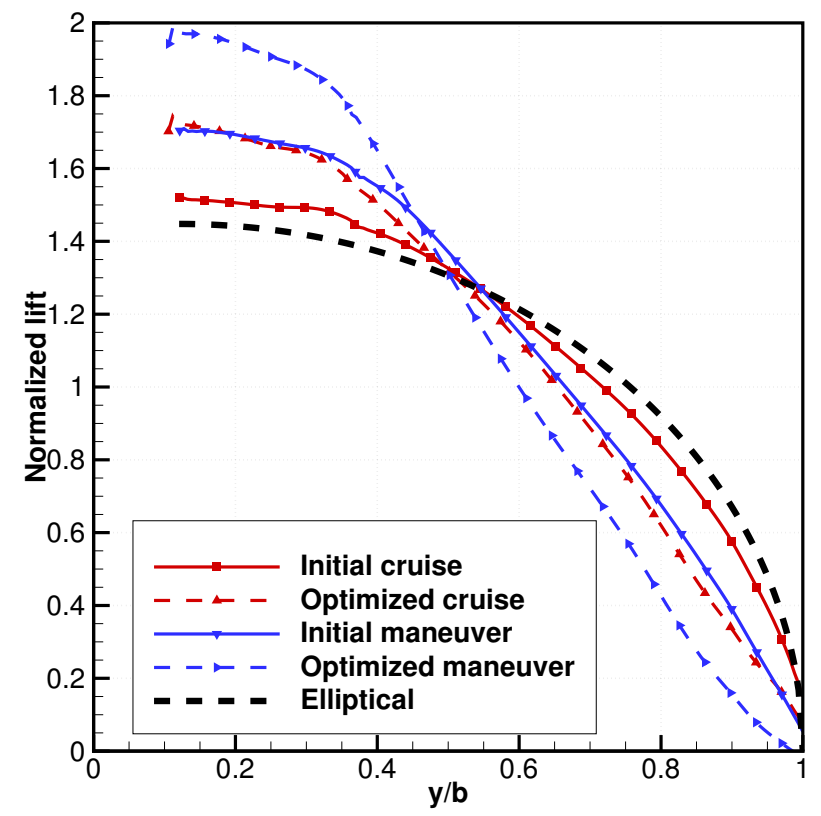

Figure 15: Span-wise lift distributions for the initial and optimized designs.

with the objective of reducing the weighted average fuel burn. The resulting aerostructural optimization contained 448 design variables and 158 constraints and solved for a total of 28 flight conditions at each iteration. Results were obtained using 931 processors in 48 hours. The coupled adjoint technique and the new kriging based methods presented in this paper allowed us to tackle an extremely large and complex design optimization problem with highfidelity methods.

We showed that a linearized form of the mission analysis is a suitable objective for a massively multi-point aerostructural optimization. The optimization results show that modifications to the configuration could obtain an $8.8 \%$ reduction in the weighted average fuel burn. The use of historical flight data ensured we optimized the aircraft 


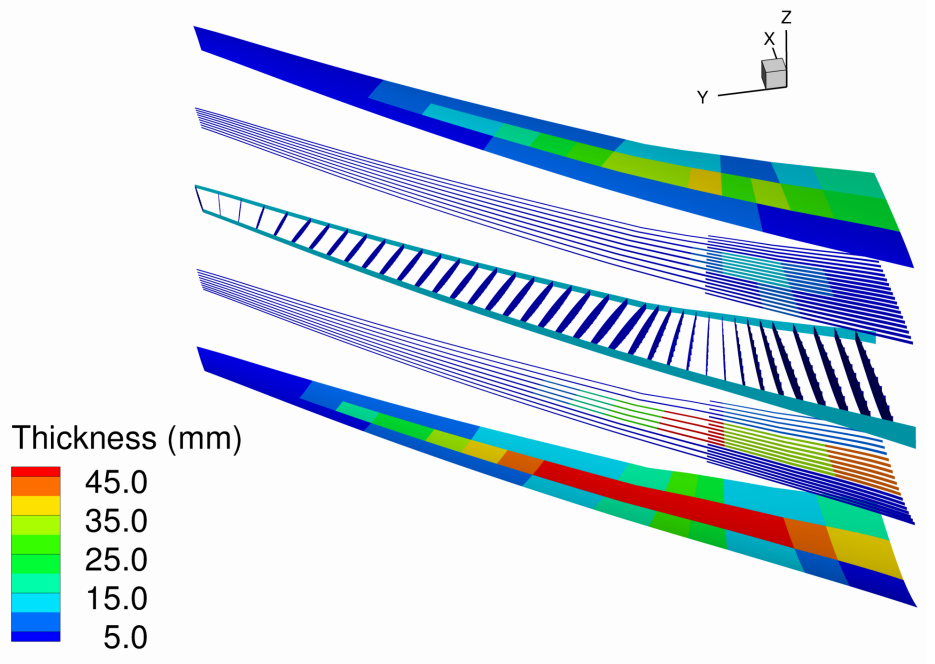

(a) Thickness distribution for initial design

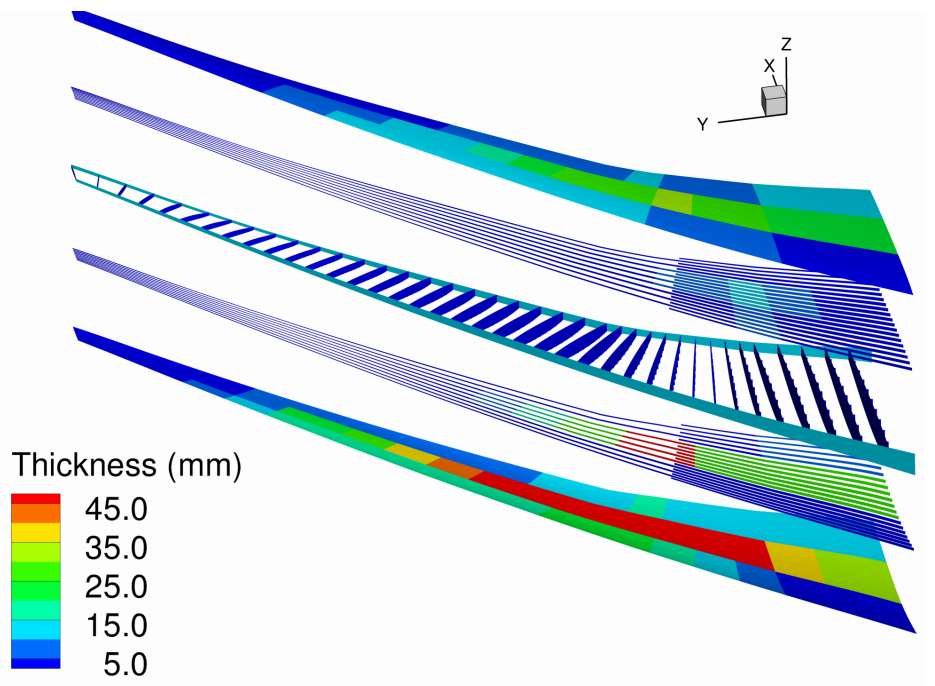

(c) Thickness distribution for optimized design

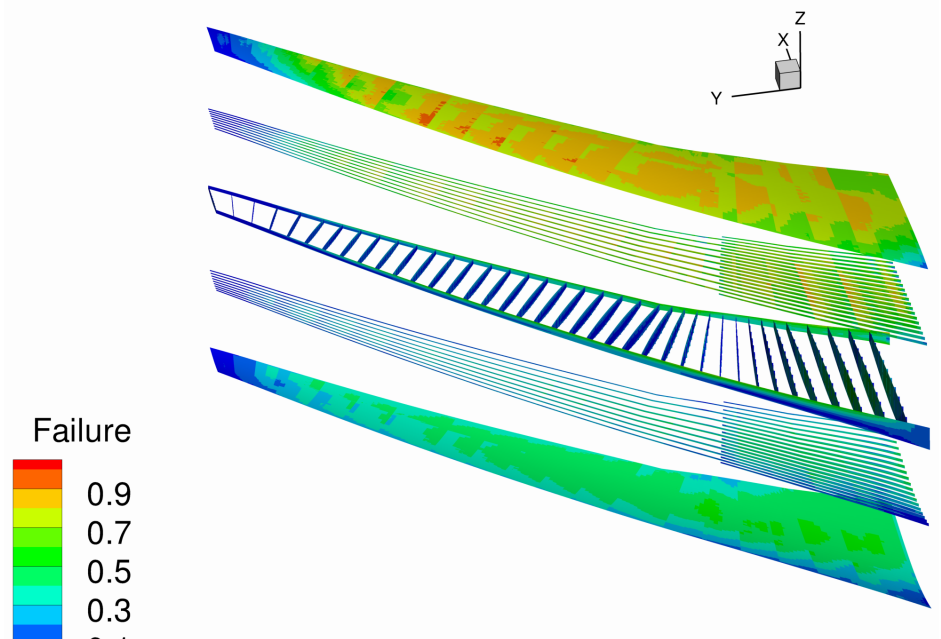

(b) Failure distribution for initial design, $2.5 \mathrm{~g}$ maneuver

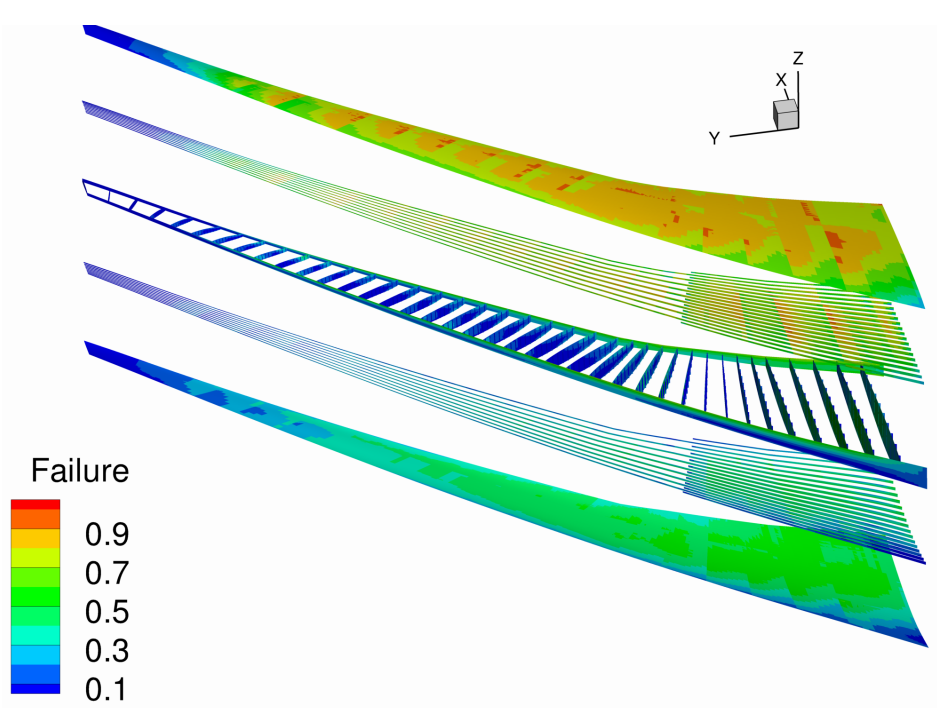

(d) Failure distribution for the optimized design, $2.5 \mathrm{~g}$ maneuver

Figure 16: Initial and optimized thickness (left column) and failure parameter (right column) distributions over the wing box.

for the set of flight conditions matching real world operations and obtained the desired trade-off between drag and weight reduction. The surrogate-model assisted multi-point optimization produced a robust design with performance improvements over the critical sections of the cruise operational envelope.

\section{Acknowledgments}

The authors are grateful for the funding provided by the Natural Sciences and Engineering Research Council. Computations were performed on the GPC supercomputer at the SciNet HPC Consortium. SciNet is funded by: the Canada Foundation for Innovation under the auspices of Compute Canada; the Government of Ontario; Ontario Research Fund - Research Excellence; and the University of Toronto.

\section{References}

[1] SciNet, University of Toronto's high performance computing facility: http://www. scinet . utoronto.ca.

[2] M.Y.M. Ahmed and N. Qin. Surrogate-Based Aerodynamics Design Optimization: Use of Surrogates in Aerodynamics 
Design Optimization. In 13th International Conference on Aerospace Science \& Aviation Technology, Cairo, Egypt, 26-28 May 2009.

[3] D. Amsallem, J. Cortial, and C. Farhat. Toward Real-Time Computational-Fluid-Dynamics-Based Aeroelastic Computations Using a Database of Reduced-Order Information. AIAA Journal, 48(9):2029-2037, September 2010.

[4] W.K. Anderson and V. Venkatakrishnan. Aerodynamic design optimization on unstructured grids with a continuous adjoint formulation. Computers \& Fluids, 28:443-480, 1999.

[5] V. Balabanov, M. Kaufman, D.K. Knill, O. Golovidov, A.A. Giunta, R.T. Haftka, B. Grossman, W.H. Mason, and L.T. Watson. Dependence of Optimal Structural Weight on Aerodynamic Shape for a High Speed Civil Transport. In 6th AIAA/USAF/NASA/ISSMO Symposium on Multidisciplinary Analysis and Optimization, Bellevue, WA, 1996. AIAA-964046-CP.

[6] J.-F.M. Barthelemy and R.T. Haftka. Approximations Concepts for Optimum Structural Design: A Review. Structural Optimization, 5(3):129-144, 1993.

[7] Boeing Commercial Airplanes. 777-200/300 airplane characteristics for airport planning. Internet, July 1998. D6-58329.

[8] H.P. Buckley, B.Y. Zhou, and D.W. Zingg. Airfoil Optimization Using Practical Aerodynamic Design Requirements. Journal of Aircraft, 47(5):1707-1719, September-October 2010.

[9] X. Cai and F. Ladeinde. A Comparison of Two POD Methods for Airfoil Design Optimization. In 35th AIAA Fluid Dynamics Conference and Exhibit, Toronto, ON, Canada, 6-9 June 2005. AIAA Paper 2005-4912.

[10] H.-S. Chung and J.J. Alonso. Design of a Low-Boom Supersonic Business Jet Using Cokriging Approximation Models. In 9th AIAA/ISSMO Symposium on Multidisciplinary Analysis and Optimization, Atlanta, GA, September 2002. AIAA Paper 2002-5598.

[11] H.-S. Chung and J.J. Alonso. Using Gradients to Construct Cokriging Approximation Models for High-Dimensional Design Optimization Problems. In 9th AIAA/ISSMO Symposium on Multidisciplinary Analysis and Optimization, Reno, NV, January 2002. AIAA Paper 2002-0317.

[12] S.E. Cliff, J.J. Reuther, D.A. Saunders, and R.M. Hicks. Single-Point and Multipoint Aerodynamic Optimization of High Speed Civil Transport. Journal of Aircraft, 38(6):997-1005, November-December 2001.

[13] M.S. Eldred, A.A. Giunta, S.S. Collis, N.A. Alexandrov, and R.M. Lewis. Second-Order Corrections for Surrogate-Based Optimization with Model Hierarchies. In 11th AIAA/ISSMO Multidisciplinary Analysis \& Optimization Conference, Albany, NY, August 30-September 1 2004. AIAA Paper 2004-4457.

[14] A.I.J. Forrester and A.J. Keane. Recent advances in surrogate-based optimization. Progress in Aerospace Sciences, 45:50-79, 2009.

[15] S.E. Gano, J.E. Renaud, J.D. Martin, and T.W. Simpson. Update strategies for kriging models used in variable fidelity optimization. Structure Multidisciplinary Optimization, 32:287-298, 2006.

[16] K.C. Giannakoglou, D.I. Papadimitriou, and I.C. Kampolis. Aerodynamic shape design using evolutionary algorithms and new gradient-assisted metamodels. Comput. Methods Appl. Mech. Engrg., 195:6312-6329, 2006.

[17] P.E. Gill, W. Murray, and M.A. Saunders. SNOPT: An SQP Algorithm for Large Scale Constrained Optimization. SIAM Review, 47(1):99-131, 2005.

[18] B. Grossman, J. Gurdal, G.J. Strauch, W.M. Eppard, and R.T. Haftka. Integrated Aerodynamic/Structural Design of a Sailplane Wing. Journal of Aircraft, 25(9):855-860, 1988.

[19] B. Grossman, R. T. Haftka, P.-J. Kao, D. M. Polen, and M. Rais-Rohani. Integrated aerodynamic-structural design of a transport wing. Journal of Aircraft, 27(12):1050-1056, 1990.

[20] R.T. Haftka. Optimization of flexible wing structures subject to strength and induced drag constraints. AIAA Journal, 15:11011106, August 1977.

[21] Z.-H. Han, S. Görtz, and R. Zimmermann. On Improving Efficiency and Accuracy of Variable-Fidelity Surrogate Modeling in Aero-data for Loads Context. In Proceeding of CEAS 2009 European Air and Space Conference, Manchester, UK, October 26-29 2009.

[22] Z.-H. Han, R. Zimmermann, and S. Görtz. A New Cokriging Method for Variable-Fidelity Surrogate Modeling of Aerodynamic Data. In 48th AIAA Aerospace Sciences Meeting Including the New Horizons Forum and Aerospace Exposition, Orlando, FL, January 4-7 2010. AIAA Paper 2010-1225.

[23] R. Hooke and T.A. Jeeves. "Direct search" solution of numerical and statistical problems. J. Assoc. Comput. Mach, 8:212-229, 1961.

[24] ICAO. Aviation and Climate Change. International Civil Aviation Organization (ICAO) Environmental Report, 2010.

[25] International Energy Agency (IEA). World Energy Outlook 2008. International Energy Agency, Paris, France, 2008.

[26] E.H. Isaaks and R.M. Srivastava. An Introduction to Applied Geostatistics. Oxford Univ. Press, Oxford, 1989.

[27] Halton. J.H. On the efficiency of certain quasi-random sequences of points in evaluating multi-dimensional integrals. $\mathrm{Nu}$ merische Mathematik, 2:84-90, 1960.

[28] R. Jin, W. Chen, and T.W. Simpson. Comparative studies of metamodelling techniques under multiple modelling criteria. Structural and Multidisciplinary Optimization, 23:1-13, 2001.

[29] Graeme J. Kennedy and Joaquim R. R. A. Martins. Parallel solution methods for aerostructural analysis and design optimization. In Proceedings of the 13th AIAA/ISSMO Multidisciplinary Analysis and Optimization Conference, Fort Worth, Texas, September 2010.

[30] Gaetan K. W. Kenway, Graeme J. Kennedy, and Joaquim R. R. A. Martins. A CAD-free approach to high-fidelity aerostructural optimization. In 13th AIAA/ISSMO Multidisciplinary Analysis and Optimization Conference, Fort Worth, TX, September 2010. AIAA-2010-9231.

[31] Gaetan K.W. Kenway, Graeme J. Kennedy, and Joaquim R. R. A. Martins. A scalable parallel approach for high-fidelity 
aerostructural analysis and optimization. In 53rd AIAA/ASME/ASCE/AHS/ASC Structures, Structural Dynamics, and Materials Conference, Honolulu, HI, April 2012. AIAA 2012-1922.

[32] P.N. Koch, T.W. Simpson, J.K. Allen, and F. Mistree. Statistical Approximations for Multidisciplinary Optimization: The Problem of Size. Special Multidisciplinary Design Optimization Issue of Journal of Aircraft, 36(1):275-286, 1999.

[33] F. Koko. Aerostructural and Trajectory Optimization of Morphing Wingtip Devices. Master's thesis, Faculty of Aerospace Engineering, Delft University of Technology, October 2011.

[34] A.B. Lambe and J.R.R.A. Martins. A unified description of mdo architectures. In Proceedings of the 9th World Congress on Structural and Multidisciplinary Optimization, Shizuoka, Japan, June 2011

[35] J. Laurenceau and M. Meaux. Comparison of gradient and response surface based optimization frameworks using adjoint method. In 4th AIAA Multidisciplinary Design Optimization Specialist Conference, Schaumburg, IL, 2008.

[36] J. Laurenceau, M. Meaux, M. Montagnac, and P. Sagaut. Comparison of gradient-based and gradient-enhanced responsesurface-based optimizers. AIAA Journal, 48(5):981-994, 2010.

[37] S. N. Lophaven, H. B. Nielsen, and J. Søndergaard. Aspects of the MATLAB toolbox DACE. Technical Report IMM-REP2002-13, Informatics and Mathematical Modelling, Technical University of Denmark, 2002.

[38] Charles A. Mader and Joaquim R. R. A. Martins. Optimal flying wings: A numerical optimization study. In 53rd AIAA/ASME/ASCE/AHS/ASC Structures, Structural Dynamics, and Materials Conference, Honolulu, HI, April 2012. AIAA 2012-.

[39] G. Matheron. Kriging, or polynomial interpolation procedures? Transactions, LXX:240-244, 1967.

[40] M. Meckesheimer, A.J. Booker, R.R. Barton, and T.W. Simpson. Computationally inexpensive metamodel assessment strategies. AIAA Journal, 40(10):2053-2060, 2002.

[41] S. Nadarajah and A. Jameson. A comparison of the continuous and discrete adjoint approach to automatic aerodynamic optimization. In AIAA 38th Aerospace Sciences Meeting and Exhibit, Reno, NV, January 2000. AIAA Paper $2000-0667$.

[42] M. Nemec, D.W. Zingg, and T.H. Pulliam. Multipoint and multi-objective aerodynamic shape optimization. AIAA Journal, 42(6):1057-1065, June 2004.

[43] AIAA Technical Committee on Multidisciplinary Design Optimization (MDO). White Paper on Current State of the Art. Technical report, Washington DC, 1991.

[44] A. Oyama, T. Nonomura, and K. Fujii. Data Mining of Pareto-Optimal Transonic Airfoil Shapes Using Proper Orthogonal Decomposition. In 19th AIAA Computational Fluid Dynamics, San Antonio, TX, 22-25 June 2009. AIAA Paper 2009-4000.

[45] Intergovernmental Panel and Climate Change (IPCC). IPCC Special Reports on Climate Change. In J.E. Penner, D.H. Lister, D.J. Griggs, D.J. Dokken, and M. McFarland, editors, Aviation and the Global Atmosphere. Cambridge University Press, UK, 1999.

[46] Nicholas M. K. Poon and Joaquim R. R. A. Martins. An adaptive approach to constraint aggregation using adjoint sensitivity analysis. Structures and Multidisciplinary Optimization, 30(1):61-73, 2007.

[47] J. Reuther, A. Jameson, J. Farmer, L. Martinelli, and D. Saunders. Aerodynamic shape optimization of complex aircraft configurations via an adjoint formulation. In 34th Aerospace Sciences Meeting and Exhibit, Reno, NV, January 1996. AIAA paper 96-0094.

[48] J. Sacks, W.J. Welch, T.J. Mitchell, and H.P. Wynn. Design and Analysis of Computer Experiments. Statistical Science, 4:409-423, 1989.

[49] J.A. Samareh. Aerodynamic shape optimization based on free-form deformation. Proc. 10th AIAA/ISSMO Multidisciplinary Anal. Optim. Conf., 6:3672-3683, 2004.

[50] S. Sgouridis, P.A. Bonnefoy, and R.J. Hansman. Air transportation in a carbon cnstrained world: Long-term dynamics of policies and strategies for mitigating the carbon footprint of commercial aviation. Transportation Research Part A: Policy and Practice, 45:1077-1091, 2011.

[51] T.W. Simpson, A.J. Booker, D. Ghosh, A.A. Giunta, P.N. Koch, and R.-J. Yang. Approximation methods in multidisciplinary analysis and optimization: a panel discussion. Struct Multidisc Optim, 27:302-313, 2004.

[52] T.W. Simpson, V. Toropov, V. Balabanov, and F.A.C. Viana. Design and Analysis of Computer Experiments in Multidisciplinary Design Optimization: A Review of How Far We Have Come-or Not. In 12th AIAA/ISSMO Multidisciplinary Analysis and Optimization Conference, Victoria, BC, Canada, September 2008. AIAA Paper 2008-5802.

[53] J. Sobieszczanski-Sobieski and R.T. Haftka. Multidisciplinary aerospace design optimization: survey of recent developments. Structural Optimization, 14:1-23, 1997.

[54] D.J.J. Toal, N.W. Bressloff, and A.J. Keane. Geometric filtration using POD for aerodynamic design optimization. In 26th AIAA Applied Aerodynamics Conference, Honolulu, HI, 18-21 August 2008.

[55] D.J.J. Toal and A.J. Keane. Efficient Multipoint Aerodynamic Design Optimization via Cokriging. Journal of Aircraft, 48(5):1685-1695, September-October 2011.

[56] V. Torczon. On the Convergence of Pattern Search Algorithms. SIAM Journal of Optimization, 7(1):1-25, February 1997.

[57] Research U.S. Department of Transportation and Bureau of Transportation Statistics Innovative Technology Administration. National Transportation Statistics: http://www.bts.gov/publications/national_transportation_ statistics/.

[58] E. van der Weide, G. Kalitzin, J. Schluter, and J.J. Alonso. Unsteady Turbomachinery Computations Using Massively Parallel Platforms. AIAA 2006-0421, 2006.

[59] John C. Vassberg, Mark A. DeHaan, S. Melissa Rivers, and Richard A. Wahls. Development of a common research model for applied CFD validation studies. In 26th AIAA Applied Aerodynamics Conference, Honolulu, Hawaii, August 2008. AIAA.

[60] G. Venter, R.T. Haftka, and Jr. Starnes, J.H. Construction of Response Surface Approximations for Design Optimization. AIAA Journal, 36(12):2242-2249, December 1998. 
[61] J.M. ver Hoef and N. Cressie. Multivariable spatial prediction. Mathematical Geology, 25(2):219-240, 1993.

[62] G.A. Wrenn. An indirect method for numerical optimization using the Kreisselmeier-Steinhauser function. NASA Technical Report CR-4220, 1989.

[63] R. Zimmermann. Asymptotic Behavior of the Likelihood Function of Covariance Matrices of Spatial Gaussian Processes. Journal of Applied Mathematics, 2010, 2010.

[64] R. Zimmermann and S. Görtz. Non-linear reduced order models for steady aerodynamics. International Conference on Computational Science, 1:165-174, 2010. 